\title{
Neonatal Oxytocin Treatment Ameliorates Autistic-Like Behaviors and Oxytocin Deficiency in Valproic Acid-Induced Rat Model of Autism
}

\begin{abstract}
Yu-Chuan Dai ${ }^{1,2,3 \dagger}$, Hong-Feng Zhang ${ }^{4 \dagger}$, Michael Schön ${ }^{5,6,7}$, Tobias M. Böckers ${ }^{5,7}$, Song-Ping Han 1,2,3,8, Ji-Sheng Han ${ }^{1,2,3}$ and Rong Zhang ${ }^{1,2,3 *}$

${ }^{1}$ Neuroscience Research Institute, Peking University, Beijing, China, ${ }^{2}$ Key Laboratory for Neuroscience, Ministry of Education, National Health and Family Planning Commission, Peking University, Beijing, China, ${ }^{3}$ Department of Neurobiology, School of Basic Medical Sciences, Peking University Health Science Center, Beijing, China, ${ }^{4}$ Fujian Provincial Key Laboratory of Neurodegenerative Disease and Aging Research, Institute of Neuroscience, College of Medicine, Collaborative Innovation Center for Brain Science, Xiamen University, Xiamen, China, ${ }^{5}$ Institute for Anatomy and Cell Biology, Ulm University, Ulm, Germany, ${ }^{6}$ International Graduate School in Molecular Medicine Ulm, Ulm University, Ulm, Germany, ${ }^{7}$ Department of Neurology, Ulm University, Ulm, Germany, ${ }^{8}$ Wuxi HANS Health Medical Technology Co., Ltd., Wuxi, China
\end{abstract}

Autism spectrum disorder (ASD) is characterized by impaired social communication and repetitive/stereotyped behaviors. The neuropeptide oxytocin (OXT) plays a critical role in regulating social behaviors in the central nervous system, as indicated in both human and animal studies. We hypothesized that central OXT deficit is one of causes of etiology of ASD, which may be responsible for the social impairments. To test our hypothesis, central OXT system was examined in valproic acid (VPA)-induced rat model of autism (VPA rat). Our results showed that adolescent VPA rats exhibited a lower level of OXT mRNA and fewer OXT-ir cells in the hypothalamus than control rats. Additionally, OXT concentration in cerebrospinal fluid (CSF) was reduced. The number of OXT-ir cells in the supraoptic nucleus (SON) of neonatal VPA rats was also lower. Autistic-like behaviors were observed in these animals as well. We found that an acute intranasal administration of exogenous OXT restored the social preference of adolescent VPA rats. Additionally, early postnatal OXT treatment had long-term effects ameliorating the social impairments and repetitive behaviors of VPA rats until adolescence. This was accompanied by an increase in OXT-ir cells. Taken together, we demonstrated there was central OXT deficiency in the VPA-induced rat model of autism, and showed evidence that early postnatal OXT treatment had a long-term therapeutic effect on the autistic-like behaviors in VPA rats.

Keywords: autism spectrum disorder, social behavior defect, repetitive behavior, exogenous supplement, oxytocin therapy

\section{INTRODUCTION}

Autism Spectrum Disorder (ASD) is a neurodevelopmental disorder characterized by impaired social interaction and communication, as well as repetitive and stereotyped behavior (Bhat et al., 2014). Although genetic factors are strongly indicated to be involved in the onset of ASD (Sebat et al., 2007; Klei et al., 2012; De Rubeis et al., 2014), they are not responsible for all cases. Specific 
environmental factors, such as prenatally viral infection (Ashwood and Van de Water, 2004; Brown, 2012) or exposure to valproic acid (VPA) (Bromley et al., 2013; Christensen et al., 2013), may increase the risk of ASD in offspring. The etiology of ASD is therefore believed to be a result of interactions between genetic and environmental factors (Hallmayer et al., 2011; Chaste and Leboyer, 2012; Tordjman et al., 2014). Due to high clinical and genetic heterogeneity (Bhat et al., 2014; Ivanov et al., 2015), the pathogenesis of ASD remains unclear, and thus the treatment of ASD is still a challenge. Clinical studies indicate that rehabilitation training can partially alleviate behavioral deficits, but they fail to rescue all of the core symptoms in individuals with ASD (Virués-Ortega, 2010; MacDonald et al., 2014; Tonge et al., 2014). Moreover, specific medication for the treatment of the social impairments or language obstacles in ASD was lacking (McCracken et al., 2002; Bowers et al., 2015). Therefore, a better understanding of the underlying mechanisms of ASD is necessary and which might provide treatment strategies in drug development.

Substantial evidence indicates that the neuropeptide oxytocin (OXT) is a powerful regulator of social behaviors (Stoop, 2012; Caldwell, 2017; Cservenak et al., 2017; Yoshihara et al., 2017). Oxytocin in the brain is synthesized and secreted by the paraventricular nucleus (PVN) and supraoptic nucleus (SON) (Stoop, 2012), and OXT-containing neural projections and OXT receptors (Oxtr) are widely distributed in the brain areas involved in regulating social behavior (Caldwell, 2017; Song and Albers, 2017). Oxt (Ferguson et al., 2000; Ferguson et al., 2001) or Oxtr (Sala et al., 2011; Pobbe et al., 2012a,b) knockout (KO) mice have been reported to exhibit less social communication and interaction, along with impaired social cognitive memory and other altered behaviors relevant to ASD (Zhang et al., 2017). Central infusion or peripheral injection of OXT has been shown to promote social approach (Lukas et al., 2011; Teng et al., 2016), pair bonding (Bales and Carter, 2003; Bales et al., 2007; Ross et al., 2009), and social memory (Popik and van Ree, 1991; Benelli et al., 1995) in rodents.

Furthermore, reversal of autistic-like behaviors following OXT administration has been observed in monogenic rodent models of autism and patients with ASD. Exogenous OXT administration was shown to rescue social impairments in mice with deletion of Oxt (Ferguson et al., 2000), Oxtr (Sala et al., 2011), Cd38 (Jin et al., 2007), CNTNAP2 (contactin-associated protein-like 2 gene) (Penagarikano et al., 2015), MAGEL2 (MAGE-like protein 2 gene) (Meziane et al., 2015), or Oprm1 (mu-type opioid receptor 1 gene) (Gigliucci et al., 2014). In clinical studies, acute OXT administration facilitated social cognition and interaction, ameliorated repetitive behavior, and enhanced language competence in patients with ASD (Hollander et al., 2003; Hollander et al., 2007; Guastella et al., 2010; Hall et al., 2012). However, the effects of repeated dosing of OXT on autistic behaviors remain ambiguous (Kosaka et al., 2012; Tachibana et al., 2013; Dadds et al., 2014; Guastella et al., 2015; Watanabe et al., 2015). Recent studies have revealed that OXT improved social cognition and social preference by enhancing social information processing (Oettl et al., 2016; Raam et al., 2017) and promoting social reward (Hung et al., 2017; Smith et al., 2017).
Although the underlying mechanisms of ASD are still largely unknown, deficiency in the OXT systems has been suggested to be involved in its development (Preti et al., 2014; Zhang et al., 2017) because of the critical role of OXT in regulating social behaviors. Deficits in OXT-immunoreactive (OXT-ir) neurons and peptide levels were observed in several monogenic mouse models of autism, including BTBR mice (Silverman et al., 2010b), CNTNAP2 (Penagarikano et al., 2015) KO mice and MAGEL2 (Meziane et al., 2015) KO mice. However, whether central OXT deficiency occurs in autistic models induced by environmental factors is unknown, as is whether OXT administration is capable of ameliorating autistic behaviors in these types of ASD models.

Valproic acid (VPA) is widely used as an antiepileptic drug in clinics (Löscher, 2002). Embryonic exposure of VPA has been reported to be associated with congenital malformations (Ornoy, 2009), cognitive impairments (Meador et al., 2013) as well as ASD (Bromley et al., 2008; Bromley et al., 2013; Smith and Brown, 2014). In animal studies, a single intraperitoneal injection of VPA during the mid-pregnancy of rats was used to produce a rat model of autism in offspring (Schneider and Przewlocki, 2005). Offspring in these cases have been reported to exhibit a series of autistic behaviors, including reduced ultrasonic communication, impaired social interaction, and repetitive/stereotyped behaviors (Roullet et al., 2013; Nicolini and Fahnestock, 2018). Importantly, this autistic model has been widely used to investigate the intrinsic link between some environmental risk factors and the pathogenesis of ASD (Stefanik et al., 2015; Bertelsen et al., 2017; Hara et al., 2017; Wu et al., 2017; Yamaguchi et al., 2017).

In the present study, the VPA-induced rat model of autism was used to investigate the relationship between its behavioral phenotype and the content of central OXT system in the brain. Changes in autistic-like behaviors in VPA rats were examined following exogenous OXT administration.

\section{MATERIALS AND METHODS}

\section{VPA-Induced Rat Model of Autism}

Male and female Wistar rats weighing 270-290 g were obtained from the Department of Experimental Animal Sciences, Peking University Health Science Center. Animals were housed individually under a regulated environment $\left(23 \pm 2^{\circ} \mathrm{C}\right.$; $50 \% \pm 10 \%$ humidity) with a 12-12 h light-dark cycle. This study was carried out following USA National Institutes of Health Guide for the Care and Use of Laboratory Animals. The protocol was approved by Peking University Animal Care and Use Committee (ethics approval ID, LA2015204).

As previously described, female and male rats were allowed to mate overnight. The day was considered embryonic day 0.5 (E0.5) in the presence of a vaginal plug. The pregnant rats were randomly distributed into two groups: VPA group and control (Ctrl) group. In VPA group, pregnant rats were intraperitoneally injected with VPA (Sigma:P4543, diluted with normal saline to a concentration of $200 \mathrm{mg} / \mathrm{ml}$ ) at a dose of $600 \mathrm{mg} / \mathrm{kg}$ body weight on E12.5 (Schneider and Przewlocki, 2005). The pregnant rats in the control group received the same volume of normal saline at 
E12.5. After weaning at postnatal day 21 (PND21), offspring of same sex were housed separately with $4-5$ per cage.

Ultimately, there were three offspring cohorts used in this study. The first cohort was used for assessment of social behaviors, anxiety, self-grooming behavior, and the immunohistochemical and biochemical analysis of central OXT system for each group (see Figure 1A for experimental procedure). Analyses were conducted on both male and female offspring in this cohort. The second cohort was used to evaluate the effect that a single intranasal OXT administration had on rat social behaviors of VPA rats at PND40. The third cohort was used to evaluate the effect of the postnatal OXT treatment (7 daily subcutaneous injections beginning from PND0) on autistic-like behaviors of VPA rats. We also analyzed the number of OXT-ir cells in the hypothalamus of VPA rats following the treatment (see Figure 6A for experimental procedure). Only male offspring were used in second and third cohorts because VPA rats had no gender differences in phenotypes (see "VPA Rats Displayed Social Impairments" and "VPA Rats Exhibited Central OXT System Deficiency")

\section{Isolation-Induced Ultrasonic Vocalizations (USVs)}

Changes in social communication of pups were tested by analyzing isolation-induced USVs as reported previously. USVs were recorded on PND7 between 18:00 and 22:00 in a quite environment with dim light. Briefly, pups were individually removed from the home cage and gently transferred to the test cage on a heating pad $\left(37^{\circ} \mathrm{C}\right)$. USVs were recorded for $300 \mathrm{~s}$ for each pup and collected by an ultrasonic microphone (CM16/CMPA, Avisoft Bioacoustics, Berlin, Germany) that hung $25 \mathrm{~cm}$ above the cage floor. The connected amplifier (AUSG$116 \mathrm{H}$, Avisoft Bioacoustics, Berlin, Germany) was set at a sampling frequency of $250 \mathrm{kHz}$ with a $125 \mathrm{kHz}$ low-pass filter. The recorded files were analyzed with Avisoft SASLab Pro (Version 4.52) using fast Fourier transform (512 FFT-length, $100 \%$ frame size, Hamming window, 50\% time-window overlap) (Xu et al., 2015).

\section{Behavioral Tests Sociability Test}

Social preference and social novelty of adolescent rats were evaluated by the three-chamber sociability test on PND 3540, as documented in past study (Zhang et al., 2015). The three-chamber apparatus comprises three rectangular plexiglass chambers $(40 \mathrm{~cm} \times 34 \mathrm{~cm} \times 24 \mathrm{~cm})$ with each side chambers connected to the central chamber by a corridor. The assay began with a 5 min habituation during which the subject rat was placed in the central chamber and allowed to freely explore the empty apparatus. Two successive stages (Stage I and Stage II) were followed. Stage I for social preference: an unfamiliar, weight and sex matched Wistar rat (Stranger 1) was locked in a wire cage and placed in one of the side chambers. An identical empty cage was placed in the other side chamber. Then the subject rat was placed in the central chamber and allowed to explore the side chambers for $10 \mathrm{~min}$ (paradigm showed in Figure 2A). Stage II for social novelty: another unfamiliar, weight and sex matched Wistar rat (Stranger 2) was placed in the empty cage used in the Stage I. The subject rat was then allowed to access to both sides freely for $10 \mathrm{~min}$ (paradigm showed in Figure 2E). During the experiment, the time spent in each of the three chambers was automatically recorded. Movement paths were video recorded and track plots were analyzed with an image video-tracking system (Smart2.5, PanLab, Harvard Apparatus, United States). To minimize the impact from residual rat odors, the entire apparatus was thoroughly cleaned with $70 \%$ ethanol at the beginning of each trial. All behavior studies were carried out during the dark cycle.

\section{Light/Dark Box Test}

The light/dark box comprised an illuminated $(40 \mathrm{~cm} \times 34 \mathrm{~cm} \times 24 \mathrm{~cm})$ and a dark $(40 \mathrm{~cm} \times 34 \mathrm{~cm} \times 24 \mathrm{~cm})$ compartment separated by a partition with a door. The test was carried out to assess the anxiety level of adolescent rats. At the beginning of the experiment, the subject was placed into the light side and allowed to explore the apparatus for $5 \mathrm{~min}$. Time spent in the light side was automatically recorded. At the beginning of each trial, the entire apparatus was thoroughly cleaned with $70 \%$ ethanol.

\section{Self-Grooming Behavior}

The self-grooming test is a paradigm that measures the level of stereotyped behavior of rodents (Kalueff et al., 2016). During habituation, animals were placed into an empty cage similar to the home cage and encouraged to explore for $10 \mathrm{~min}$. Rat behavior was then video-taped for $10 \mathrm{~min}$ and the total self-grooming time was calculated. In the present study, selfgrooming behaviors include: (1) wiping the nose, face, head, and ears with forepaws; (2) licking body, anogenital area, and tail (Kalueff et al., 2016).

\section{OXT Treatment}

\section{Single Intranasal Treatment}

To verify the pro-social effect of OXT treatment in adolescent VPA rats, a single dose of OXT (ProSpec-Tany TechnoGene Ltd, Rehovot, Israel) or normal saline was administered intranasally in control and VPA rats $30 \mathrm{~min}$ before the social preference test. OXT was dissolved in normal saline to a concentration of $1 \mu \mathrm{g} / \mu \mathrm{l}$. Gently and fleetingly, $20 \mu \mathrm{l}$ of saline or OXT solution was applied bilaterally on the rhinarium with a P10 pipette. The rat was held with its nose up for $1 \mathrm{~min}$ to ensure that liquid was inhaled and diffused into nostrils thoroughly (Lukas and Neumann, 2012; Neumann et al., 2013). Rats were then released to their home cage until the test started.

\section{Repeated Subcutaneous Dosing at an Early Postnatal Stage}

To achieve long-term therapeutic effect on social behaviors, we attempted to administer OXT at an early postnatal stage since it had been suggested to have long-term effects on social behaviors in other rodent models of autism. Within $24 \mathrm{~h}$ of birth, VPA pups received randomly either a single subcutaneous injection of OXT $(3 \mu \mathrm{g} / 20 \mu \mathrm{l}$ in normal saline) or $20 \mu \mathrm{l}$ normal saline. Subsequently, an identical dose of OXT solution or normal saline 
was administered daily in the following 6 days (Meziane et al., 2015). Control pups only received equal volume of normal saline during the first week after birth. Isolation-induced USVs of rat pups was evaluated on PND7. After weaning, offspring from same litter were housed together (3-4 pups per cage) and were subjected to the rest of experiments from PDN35 to PND40 (see Figure 6A for experiment procedure).

\section{Tissue Collection}

For determining OXT mRNA and peptide levels, rats were anesthetized by intraperitoneal injection with $10 \%$ chloral hydrate $(300 \mathrm{mg} / \mathrm{kg}$ body weight) before sampling.

\section{Cerebrospinal Fluid (CSF) Collection}

Under general anesthesia, rat heads were immobilized in a stereotaxic frame (David Kopf Instruments, CA, United States) in the flat-skull position. After incision of the skin on the back of neck and blunt dissection of dorsal muscles, the Cerebrospinal Fluid (CSF) $(\sim 150 \mu \mathrm{l})$ was collected from the cisterna magna. All $\mathrm{CSF}$ samples were stored at $-80^{\circ} \mathrm{C}$ until assayed. According to our preliminary experiments, $450 \mu \mathrm{l}$ of CSF was required to assess OXT levels using an oxytocin ELISA kit (see below). Therefore, every three CSF samples of the same group and of the same sex were pooled and analyzed as a single sample. Because the number of CSF samples was limited, the data from both sex were analyzed together.

\section{Blood-Sample Collection}

Following decapitation, trunk blood $(\sim 5 \mathrm{ml})$ was collected rapidly into a clean tube containing Aprotinin (Sigma-Aldrich, United States) at a final concentration of $500 \mathrm{KIU} / \mathrm{ml}$ of blood. The blood samples were maintained at room temperature for $30 \mathrm{~min}$, and then centrifuged at $1600 \mathrm{~g}$ for $15 \mathrm{~min}$ at $4^{\circ} \mathrm{C}$. The serum was separated and divided into aliquots of $650 \mu \mathrm{l}$ and stored at $-80^{\circ} \mathrm{C}$.

\section{Brain-Tissue Collection}

Rats were decapitated and the brain was quickly removed and frozen in liquid nitrogen for $20 \mathrm{~s}$. The frozen brains were stored at $-80^{\circ} \mathrm{C}$ until the PVN and SON were dissected from the hypothalamus. According to the Paxinos and Watson Rat Brain Atlas, bilateral punches of the PVN and SON (1 mm and $1.5 \mathrm{~mm}$ in diameter, respectively) were taken at $-20^{\circ} \mathrm{C}$ and then store at $-80^{\circ} \mathrm{C}$ until assayed.

\section{Determination of OXT mRNA Levels}

Total mRNA in unilateral punches of PVN or SON was extracted with TRIzol reagent (Invitrogen, Carlsbad, CA, United States) according to the manufacturer's instructions and digested with DNase (Promega, Madison, WI, United States) to remove DNA contamination. For cDNA synthesis, reverse-transcription of the mRNA was performed with the PrimeScript RT-PCR kit (TaKaRa, Dalian, China). Gene expression analysis of OXT and the house-keeping gene ( $\beta$-actin) was evaluated using TaqMan Gene Expression Assays (assay ID: OXT, Rn00564446_g1; $\beta$-actin, Rn00667869_m1) following standard qPCR procedure: $2 \mathrm{~min}$ at $50^{\circ} \mathrm{C}, 10 \mathrm{~min}$ at $95^{\circ} \mathrm{C}, 40$ cycles of $15 \mathrm{~s}$ at $95^{\circ} \mathrm{C}$ and $1 \mathrm{~min}$ at $60^{\circ} \mathrm{C}$. The results were calculated using the $2^{-\Delta \Delta \mathrm{CT}}$ method with $\beta$-actin as the endogenous reference gene, and ultimately converted to fold changes versus control.

\section{Detection of OXT Levels in the CSF and Serum}

OXT content in the CSF and serum was assayed using a commercially available ELISA kit (Enzo Life Sciences, PA, United States). All samples were subjected to prior extraction with acetone and petroleum ether according to the product manual. The ELISA kit was highly sensitive (detecting limit: 15.0 $\mathrm{pg} / \mathrm{ml}$ for OXT) with little cross-reactivity (less than $7.5 \%$ ) with $\mathrm{arg}^{8}$-vasopressin. The intra-assay CV for OXT was $10.2 \%$.

\section{Immunohistochemistry}

Rats were anesthetized by intraperitoneal injection with $10 \%$ chloral hydrate $(300 \mathrm{mg} / \mathrm{kg}$ body weight). They were then transcardially perfused with normal saline $\left(37^{\circ} \mathrm{C}\right)$ and $4 \%$ paraformaldehyde in $0.1 \mathrm{M}$ phosphate buffer $\left(\mathrm{PFA}, 4^{\circ} \mathrm{C}, \mathrm{pH}\right.$ 7.4). Brains were quickly removed and post-fixed in $4 \%$ PFA at $4{ }^{\circ} \mathrm{C}$ for $12 \mathrm{~h}$. Brains were sequentially soaked in 20 and $30 \%$ sucrose in $0.1 \mathrm{M}$ phosphate buffer until saturated for cryoprotection. Each brain was coronally sectioned into $30-\mu \mathrm{m}$ thick slices (20 $\mu \mathrm{m}$ for rat pups) using a freezing microtome and sections were then stored at $-20^{\circ} \mathrm{C}$ in anti-freezing solution. OXT staining was performed by the modified ABC (avidin-biotinperoxidase complex) immunohistochemical technique. Before each stage of the process, sections were rinsed in $0.01 \mathrm{M}$ phosphate buffered saline (PBS, pH 7.2) three times (5 min each). The procedures were performed as follows: brain sections were fixed in $4 \%$ PFA for $10 \mathrm{~min}$ and then, pre-incubated in $0.3 \%$ hydrogen dioxide for $30 \mathrm{~min}$ at $37^{\circ} \mathrm{C}$ to eliminate endogenous peroxidase activity. Thereafter, the sections were incubated with $10 \%$ normal goat serum and $0.3 \%$ Triton X-100 in $0.01 \mathrm{M}$ PBS for $30 \mathrm{~min}$ at $37^{\circ} \mathrm{C}$, followed by incubation with rabbit antiOXT antibody (Abcam, United States., 1:2000 dilution in 0.01 M PBS containing $1 \%$ normal goat serum and $0.3 \%$ TritonX$100)$ at $4^{\circ} \mathrm{C}$ for $48 \mathrm{~h}$. The sections were then incubated in the anti-rabbit secondary immunoglobulin G (IgG) conjugated to horseradish peroxidase (OriGene Technologies, Inc., Beijing, China) at $37^{\circ} \mathrm{C}$ for $30 \mathrm{~min}$. Immunoreactivity was visualized using the 3,3'-diaminobenzidine kit (OriGene Technologies, Inc., Beijing, China) to produce a brown nuclear reaction product. Lastly, the free-floating sections were mounted onto gelatin coated glass slides, dehydrated in a series of alcohols, cleared in xylene, and placed under a coverslip with gum.

Six sections containing the PVN or SON (between -0.8 and $2.1 \mathrm{~mm}$ from the Bregma) were selected every $150 \mu \mathrm{m}$ for OXT immunohistochemical analysis. The total number of OXT-ir cells in all six sections was counted as the data for each sample. OXT-ir cells were photographed bilaterally in the PVN and SON under a Leica automated DMI-inverted microscope (Leica DMI 4000B). Coronal sections were matched between samples.

\section{Statistics}

The number of animal used in each experiment is mentioned in figure legends. Statistical analyses were performed and graphs were generated by GraphPad Prism 7 (GraphPad Software Inc., San Diego, CA, United States). All results are expressed as 


\section{A Timeline of behavioral tests for VPA rats}

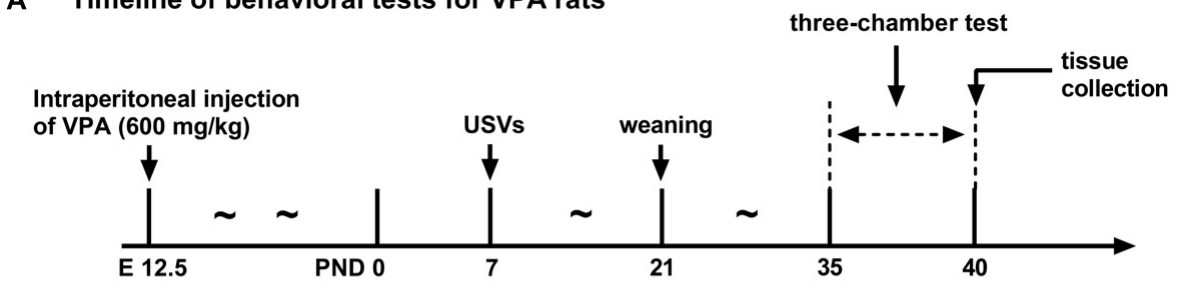

B
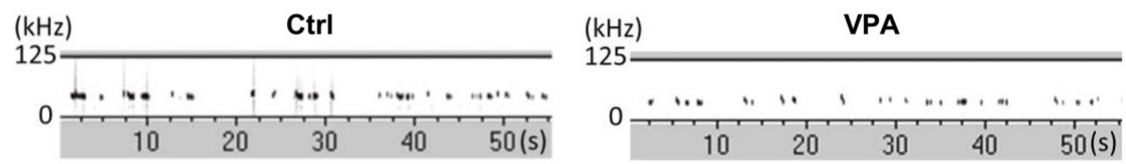

C
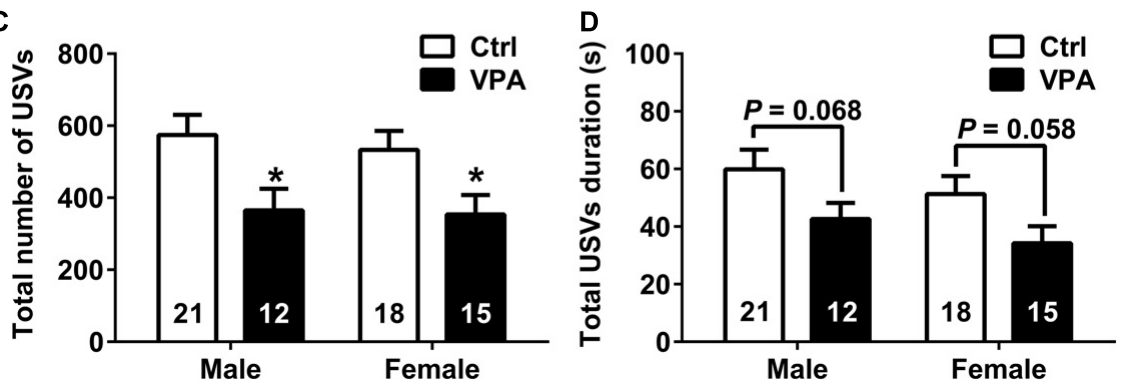

FIGURE 1 | Timeline of behavioral tests and USVs analysis of control and VPA rats. (A) Experimental design for behavioral tests. (B) Representative spectrograms of USVs recorded from control and VPA pups. (C) Both male $(n=12)$ and female $(n=15)$ rats in VPA group emitted fewer USVs in 5 min compared with control group (male $n=21$, female $n=18$ ). (D) Total USVs duration tended to be shorter in the VPA group of both sexes. Data are expressed as mean $\pm \mathrm{SEM},{ }^{*} P<0.05$. Ctrl, control; USVs, ultrasonic vocalizations.

mean \pm SEM. Unpaired Student's $t$ test or Mann-Whitney $\mathrm{U}$ test was used for behavioral and biochemical comparisons between groups (VPA versus control) or treatment (normal saline versus OXT). The three-chamber social test was analyzed with paired $t$ test to determine within-group side preference. Two-way ANOVA followed by a Tukey post hoc test was used to correct for multiple comparisons. Statistical significance was set at $P<0.05$ (two-tailed) in all tests.

\section{RESULTS}

\section{VPA Rats Displayed Social Impairments}

Social deficits of VPA rats were assessed by USVs and threechamber sociability test (see Figure 1A for experiment procedure). Results of isolation-induced USVs test (representative USVs spectrograms showed in Figure 1B) showed that the VPA group emitted fewer USVs than the control group (unpaired $t$ test; male $P<0.05$, female $P<0.05$, Figure 1C). Additionally, we observed a trend toward differences in total USVs duration between the VPA pups and control pups (unpaired $t$ test; male $P=0.068$, female $P=0.058$, Figure 1D). These data indicated a social communication impairment in infant of VPA rats.

In three-chamber sociability test (social preference, see illustration in Figure 2A and representative movement traces in
Figure 2B), the control rats spent more time in the side with Stranger 1 than in the empty cage (paired $t$ test; male $P<0.001$, female $P<0.001$, Figure 2C). In contrast, VPA rats seemed to lose interest in social interaction with conspecifics, spending a comparable time exploring the cage containing Stranger 1 rat and the empty cage (paired $t$ test; male $P=0.4699$, female $P=0.2082$, Figure 2C). Furthermore, the social sniffing time was significantly lower in VPA rats than in controls (unpaired $t$ test; male $P<0.001$, female $P<0.01$, Figure $2 \mathrm{D}$ ). These data indicated that social interaction was impaired in adolescence of VPA rats. In contrast, both VPA male and female rats exhibited normal social novelty (see illustration in Figure 2E), as they spent more time in proximity to the Stranger 2 rat than the Stranger 1 rat (paired $t$ test; male $P<0.01$, female $P<0.05$, Figure $2 \mathrm{~F}$ ).

\section{VPA Rats Exhibited Central OXT System Deficiency}

In infancy, the number of OXT-ir cells in SON was lower in VPA pups than in control pups for both males and females (representative images of OXT staining showed in Figures 3A,B; unpaired $t$ test; male $P<0.05$, female $P<0.01$, Figure 3D). In the PVN, the number of OXT-ir cells in male VPA pups was fewer than in male controls. In contrast, the females did not display any significant differences (male $P=0.05$, female $P=0.3560$, Figure 3C). 
A

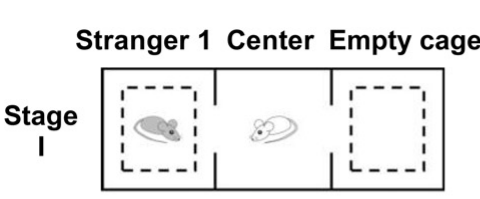

B Stranger 1 Center Empty cage

Ctrl

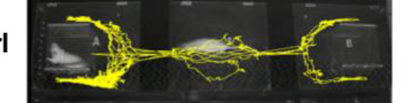

VPA

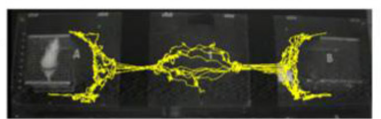

C

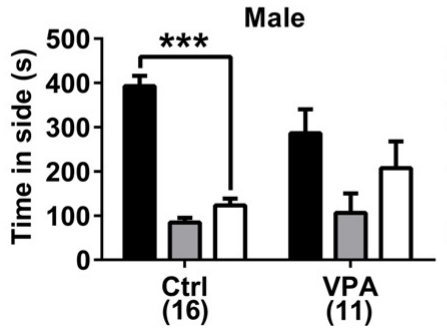

D
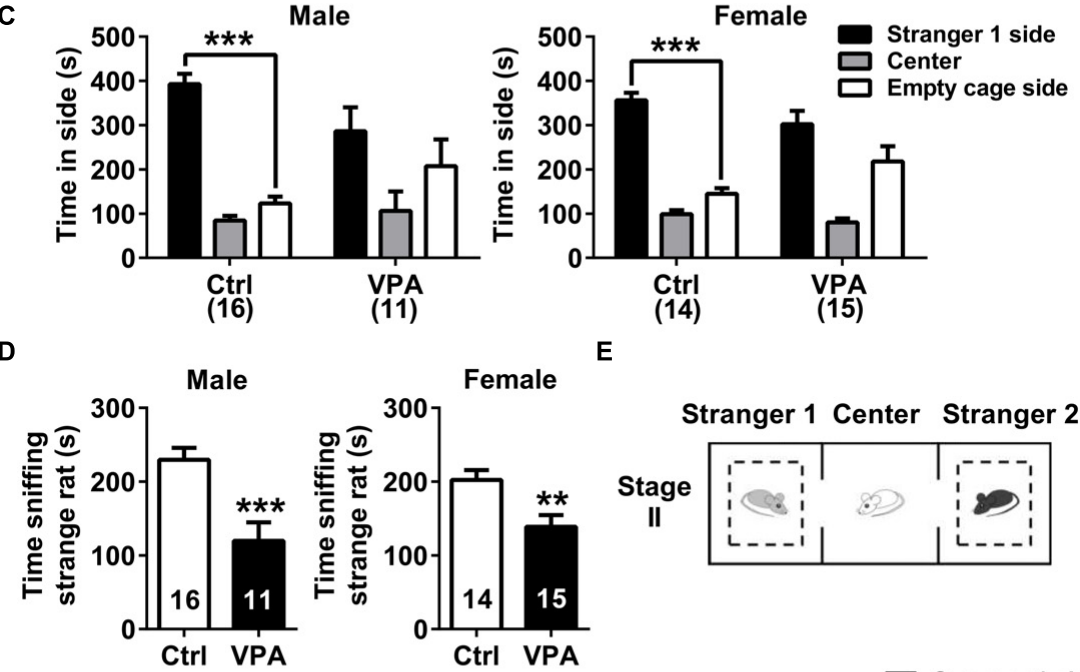

E

F

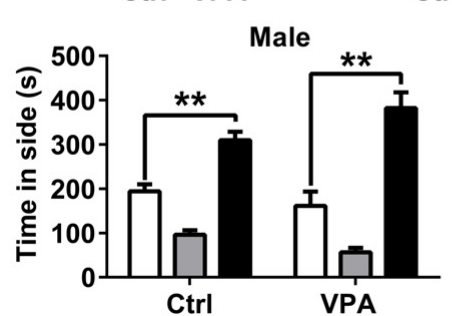

(16)

(11)

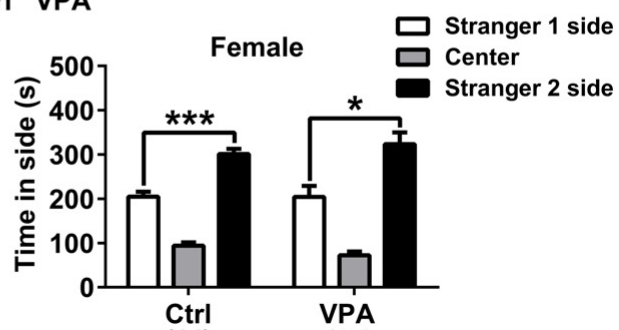

(14) (15)

FIGURE 2 | Social interaction in control and VPA rats. (A-D) Stage I of three-chamber sociability test (social preference). (A) The paradigm for testing social preference. (B) Representative traces from control and VPA rats exploring the two sides (Stranger 1 and empty cage). Both the males $(n=11)$ and females $(n=15)$ in the VPA group exhibited deficits in social preference (C) and spent less time sniffing the stranger (D) than the control group (male $n=16$, female $n=14)$. (E,F) Stage II of three-chamber sociability test (social novelty). (E) The paradigm for testing social novelty. (F) No differences were observed between VPA and control rats of both sexes in the social novelty test. Data are expressed as mean $\pm \mathrm{SEM},{ }^{*} P<0.05,{ }^{* *} P<0.01,{ }^{* * *} P<0.001$. Ctrl, control.

OXT mRNA levels were significantly lower in the SON of male and female VPA rats (unpaired $t$ test; male $P<0.001$, female $P<0.001$, Figure 4B), but not in the PVN (male $P=0.2153$, female $P=0.4350$, Figure 4A). OXT levels in the CSF of VPA rats were also lower than in control rats (unpaired $t$ test, $P<0.05$, Figure 4C). In contrast, serum OXT levels showed no significant differences between VPA and control rats in either sex (unpaired $t$ test; male $P=0.0542$, female $P=0.1968$, Figure 4D). In summary, by using immunohistochemical, RT-PCR and ELISA analyses, we have shown central OXT deficiency in VPA rats.

\section{Effect of Acute OXT Treatment on Social Behavior}

Sociability of adolescent VPA rats was assessed using the three chamber sociability test $30 \mathrm{~min}$ after the intranasal OXT treatment. The results showed that impaired sociability in
VPA rats was partially reversed following the OXT treatment, characterized by spending more time in the side with Stranger 1 than with the empty cage (see representative movement traces in Figure 5A; paired $t$ test for Stranger 1 vs. empty cage, $\mathrm{VPA}+\mathrm{OXT} P<0.01$, Figure 5B), and by spending more time in social sniffing (VPA + OXT vs. VPA + NS, $P<0.05$, Figure $5 \mathrm{C}$ ).

\section{Early Postnatal OXT Treatment Exhibited Long-Term Behavioral Effect}

For testing the therapeutic effect of early OXT treatment on the VPA rats, postnatal OXT supplement was administrated to neonate VPA rats and then sociability, self-grooming and anxiety were evaluated during adolescence (see Figure 6A for experiment procedure).

Analysis showed that early postnatal OXT treatment had a tendency to improve the total number of USVs for VPA 
A

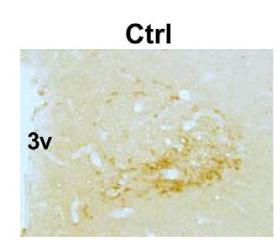

PVN

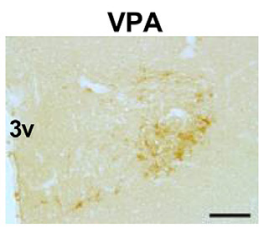

B

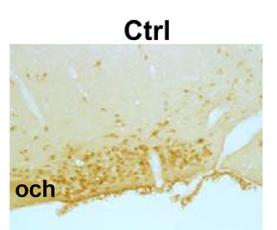

SON

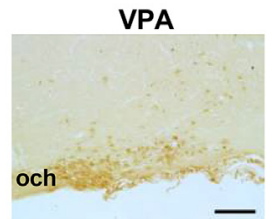

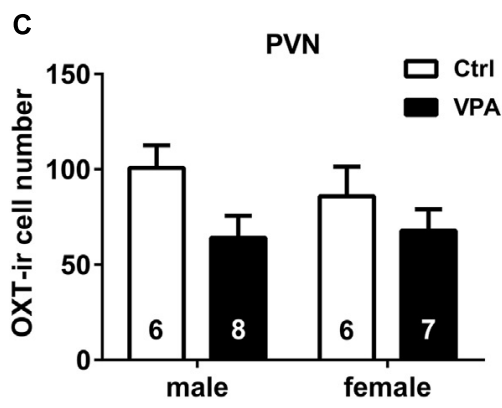

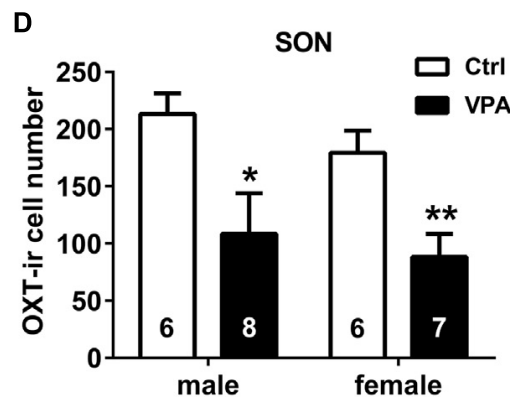

FIGURE 3 | Neonatal VPA rats had fewer OXT-ir cells in the hypothalamus than controls. Representative images (bar $=100 \mu \mathrm{m})$ of OXT staining in the PVN (A) and SON (B) of both control and VPA rats. In the PVN (C), the number of OXT-ir cells did not differ between groups. But in the SON (D), they were less in the VPA rats than controls. Data are expressed as mean \pm SEM (control: male $n=6$, female $n=6$; VPA: male $n=8$, female $n=7$ ), ${ }^{*} P<0.05,{ }^{* *} P<0.01$. Ctrl, control; PVN, paraventricular nucleus; SON, supraoptic nucleus; $3 v$, third ventricle; and och, optic chiasm.

A

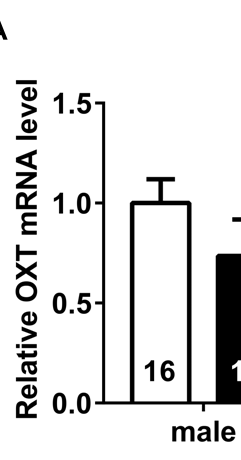

C

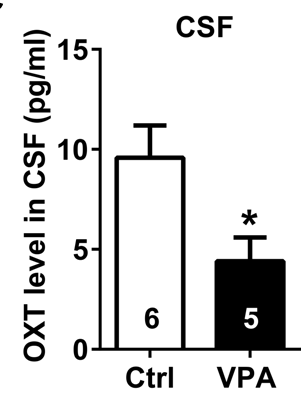

PVN
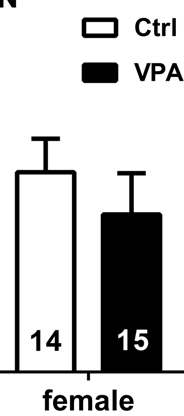

B

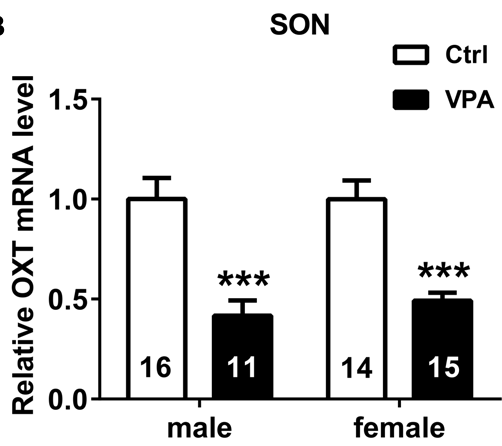

D

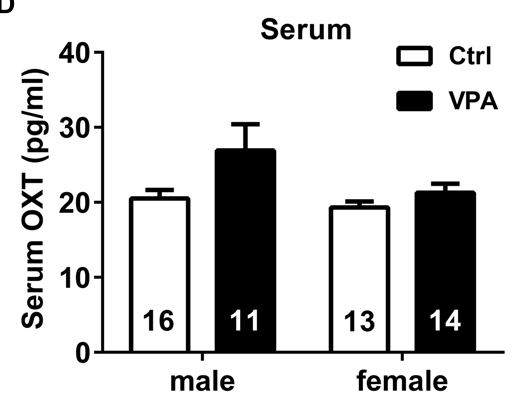

FIGURE 4 | Adolescent VPA rats exhibited deficient central OXT mRNA and peptide levels. (A,B) Compared to control rats (male $n=16$, female $n=14$ ), lower OXT mRNA levels were observed in the SON, but not in the PVN of male $(n=11)$ and female $(n=15)$ VPA rats. (C) OXT levels in the CSF were also lower in VPA rats (control $n=6$, VPA $n=5$ ). (D) Serum OXT levels did not differ significantly between control (male $n=16$, female $n=13$ ) and VPA (male $n=11$, female $n=14$ ) rats Data are expressed as mean $\pm \mathrm{SEM},{ }^{*} P<0.05,{ }^{* * *} P<0.001$. Ctrl, control; PVN, paraventricular nucleus; SON, supraoptic nucleus; and CSF, cerebrospinal fluid.

pups (representative USVs spectrograms showed in Figure 6B; unpaired $t$ test; $\mathrm{Ctrl}+\mathrm{NS}$ vs. VPA $+\mathrm{NS} P<0.01$, VPA + OXT vs. $\mathrm{VPA}+\mathrm{NS} P=0.0764$, Figure 6C). Additionally, we observed that chronic OXT treatment in the early stage of life rescued in certain degree some of the social deficits found in adolescent VPA rats in three-chamber sociability test (see representative movement 

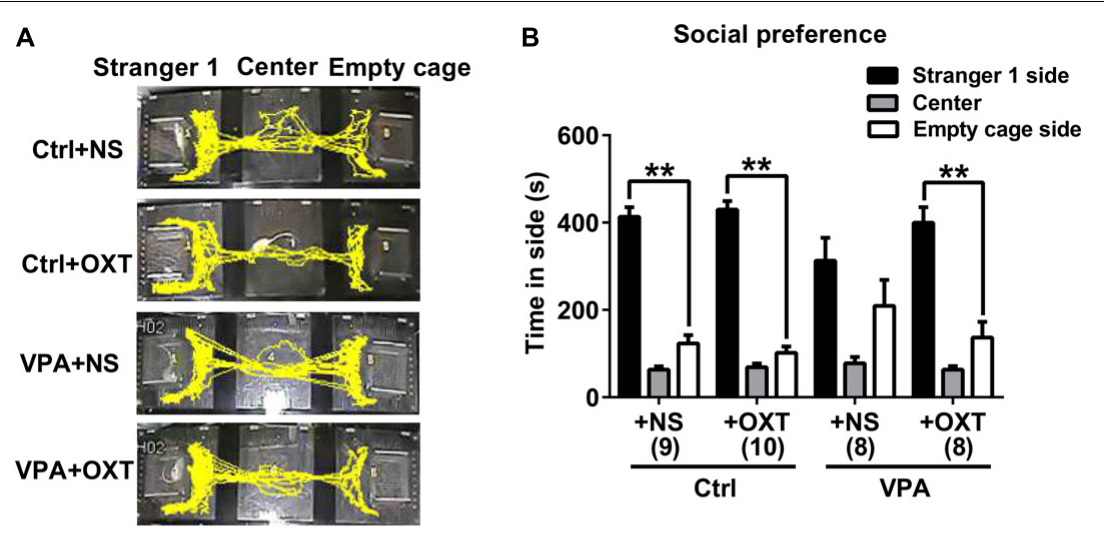

C

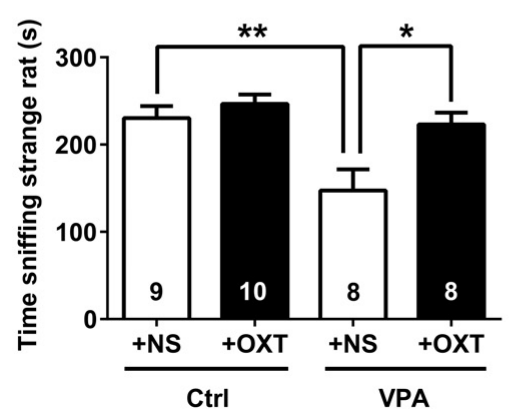

FIGURE 5| Acute OXT administration ameliorated social deficits of VPA rats. (A) Representative traces from the three-chamber social preference test for control and VPA rats treated with normal saline (NS) or OXT. (B,C) A single dose of intranasal OXT treatment on VPA rats improved their social preference (B) and prolonged the time spent sniffing conspecifics (C). Data are expressed as mean \pm SEM (Ctrl + NS $n=9$, Ctrl + OXT $n=10$, VPA + NS $n=8$, VPA + NS $n=8),{ }^{*} P<0.05$,

${ }^{* *} P<0.01$. Ctrl, control.

A

Neonatal OXT treatment

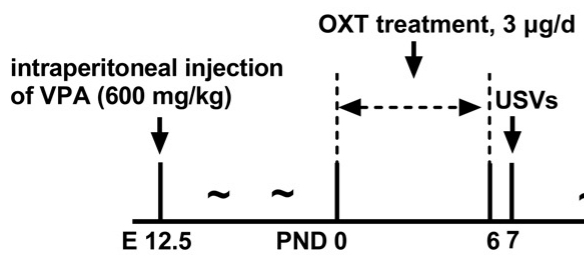

B

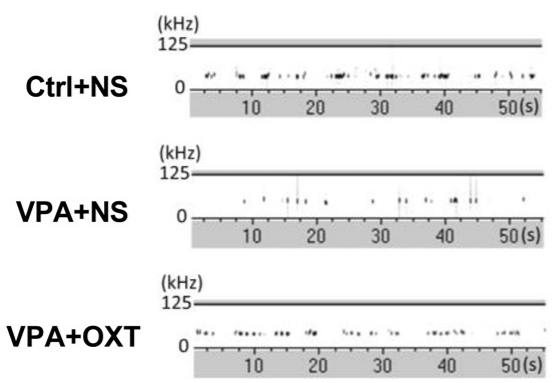

1. three-chamber test

2. self-grooming test

3. light-dark box test

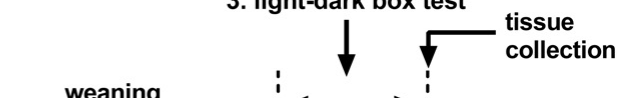

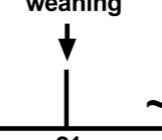

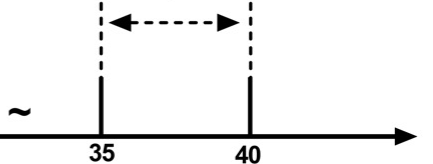

C

USVs

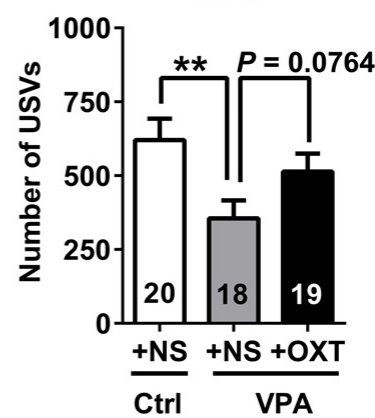

FIGURE 6 | Early postnatal OXT administration had a tendency to improve the number of USVs emitted by VPA pups. (A) Experimental design for measuring the effect of neonatal OXT treatment. (B,C) Representative USV spectrogram recorded from rat pups (B) and total number of USVs (C). Data are expressed as mean \pm SEM (Ctrl + NS $n=20$, VPA + NS $n=18$, VPA + OXT $n=19)$, **P $<0.01$. Ctrl: control; USVs, ultrasonic vocalizations. 


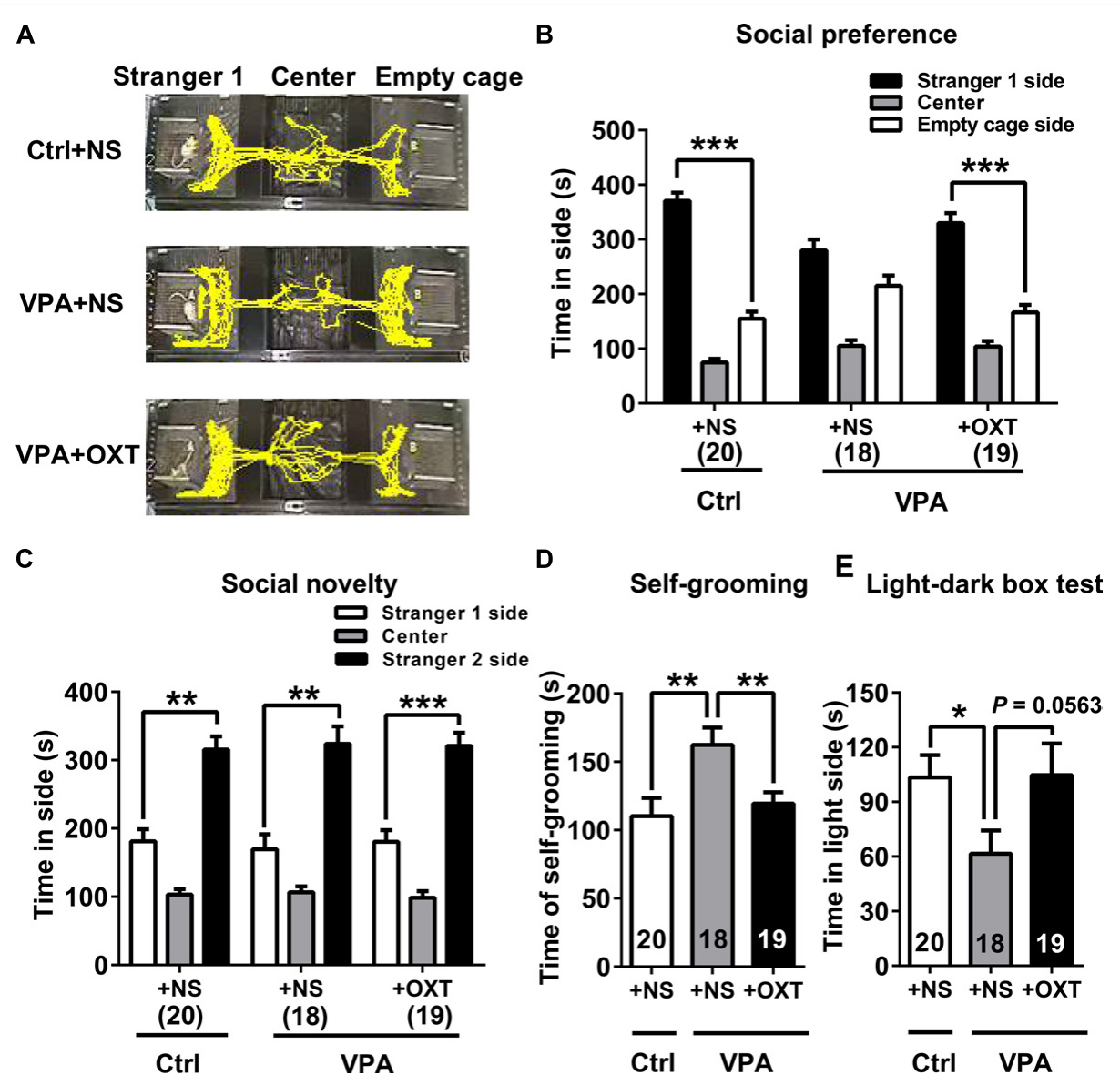

FIGURE 7 | Early postnatal OXT administration had a long-term therapeutic effect on autistic behaviors of VPA rats. (A,B) Social preference of VPA rats was normal following early postnatal OXT treatment. (C) Social novelty of VPA rats was not affected. (D) Time spent in self-grooming by VPA rats was lowered following early postnatal OXT administration. (E) The time in the light side of light-dark box was not significantly different following OXT treatment during the light-dark box test. Data are expressed as mean \pm SEM (Ctrl $+\mathrm{NS} n=20, \mathrm{VPA}+\mathrm{NS} n=18, \mathrm{VPA}+\mathrm{OXT} n=19),{ }^{*} P<0.05,{ }^{* *} P<0.01,{ }^{* * *} P<0.001 . \mathrm{Ctrl}, \mathrm{control}$

traces in Figure 7A). Adolescent rats in the VPA + OXT group spent more time exploring around the Strange 1 than the empty cage $(P<0.001$, Figure $7 \mathbf{B})$, indicating a normal social preference. For social novelty, rats in VPA + OXT group spent more time near the Strange 2 than the Stranger 1, suggesting that early postnatal OXT treatment had no influence on social novelty in VPA rats (Figure 7C).

In the present study, the duration of self-grooming was significantly longer in VPA rats than in controls (unpaired $t$ test for VPA + NS vs. Ctrl + NS, $P<0.01$, Figure 7D). The light-dark box test (Figure 7E) revealed that VPA rats spent significantly less time in the light side than control rats did (unpaired $t$ test VPA + NS vs. Ctrl + NS, $P<0.05$ ), indicating a higher level of anxiety in VPA rats. Intriguingly, early postnatal intervention of OXT significantly reduced the time that adolescent VPA rats spent in self-grooming (unpaired $t$ test for VPA + OXT vs. VPA + NS, $P<0.01$, Figure 7D). Further, their anxiety levels also tended to be lower following OXT treatment, although with marginal significance (unpaired $t$ test for VPA + OXT vs. VPA + NS, $P=0.0563$, Figure 7E).

\section{Effect of Early Postnatal OXT Treatment on OXT-ir Cell Numbers}

In adolescence, the number of OXT-ir cells in both the PVN and SON of VPA rats were significantly less than in control rats (representative images of OXT staining showed in Figures 8A,B; unpaired $t$ test for VPA + NS vs. Ctrl + NS, PVN $P<0.01$, SON $P<0.05$, Figures 8C,D respectively). Analysis showed that the numbers of OXT-ir cells were significantly restored in the PVN of VPA rats following postnatal OXT treatment (unpaired $t$ test for VPA + OXT vs. VPA + NS, $P<0.01$, Figure 8C). A similar trend was seen in the SON, however, the increase in OXT-ir cells following OXT treatment did not reach statistical significance (unpaired $t$ test for VPA + OXT vs. VPA $+\mathrm{NS}, P=0.1740$, Figure 8D).

\section{DISCUSSION}

Because of the close relationship between OXT and social behavior, we examined the role of OXT in the VPA-induced rat model of autism. Through a series of behavioral tests, we 


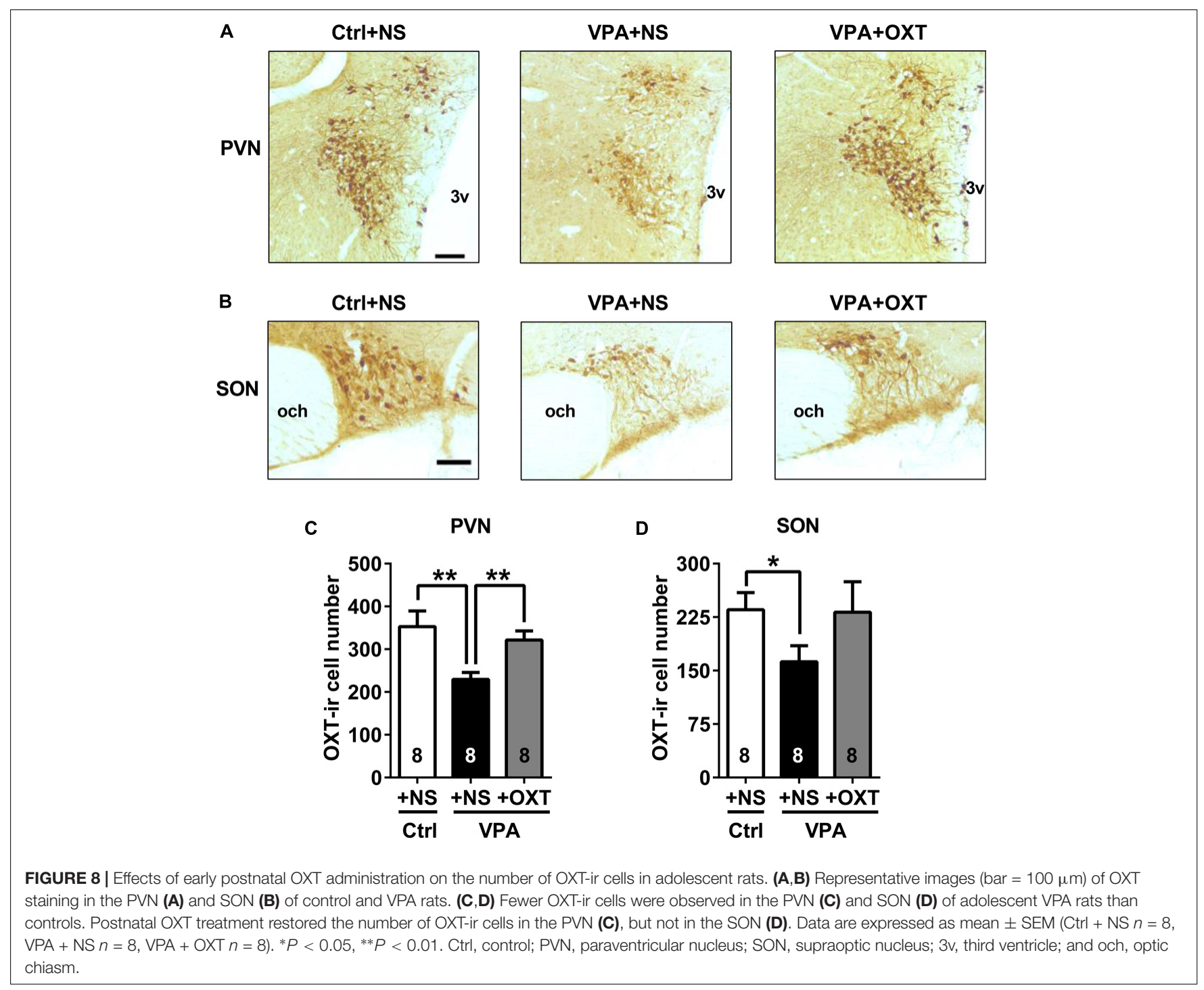

confirmed autistic-like behaviors in VPA rats. Here, we report for the first time that central OXT system is impaired in the VPA rats. Compared to controls, mRNA level and peptide concentration in both male and female VPA rats were lower in the hypothalamus. The peptide level was also decreased in CSF. OXT-ir cells were also deficient in the hypothalamus of VPA treated neonates and adolescents. Additionally, acute intranasal OXT administration improved the impaired social behavior of adolescent VPA rats. Another finding is that early neonatal OXT manipulation had a long-term therapeutic effect on autistic-like behaviors in VPA rats, accompanied by restoration of OXT-ir cells in the hypothalamus. Our results hinted that OXT plays a crucial role in the pathogenesis of social deficits in VPA rats.

ASD is a typical male-biased neurodevelopmental disorder, with a male-to-female ratio of approximately 4 to 1 (Constantino and Charman, 2012; VanderLaan et al., 2015). In contrast, our study showed that both male and female VPA rats displayed similar degree of social deficits, indicating no sex bias for this rodent autistic model. This is in accordance with clinical epidemiological findings that the sex ratio of childhood autism was 1.2 (boys to girls) for children exposed to VPA in utero (Rasalam et al., 2005; Christensen et al., 2013). These findings indicated that prenatal exposure to VPA may affect the male and female offspring similarly.

The three-chamber sociability test indicated that the social preference of VPA rats was impaired, while their social novelty was normal. To a certain extent, the latter behavior reflects the ability of animals for social recognition (Silverman et al., 2010a; van der Kooij and Sandi, 2012). The results indicated that VPA rats had normal social recognition and social memory. Our findings are supported by a previous study in which the VPA exposure at the same dose (600 $\mathrm{mg} / \mathrm{kg}$ body weight) on E12.5 led to abnormal social preference and social communication, but normal social novelty in mice (Moldrich et al., 2013). This suggests that social interaction and social memory may be two relatively independent social behaviors with different level of tolerability to environmental changes. In addition, one type of behavioral impairment does not affect the other. 
Central OXT deficiency in VPA rats might result from pharmacological action of VPA. VPA is a histone deacetylase (HDAC) inhibitor (Kataoka et al., 2013; Moldrich et al., 2013) and can increase acetylation of histone $\mathrm{H} 4$ in cultured cells in a dose-dependent manner (Gottlicher et al., 2001) Changes in histone acetylation can affect chromatin structure reorganization and interfere with gene expression. Similarly, in utero exposure to VPA resulted in an increased level of acetylated histones in mouse embryo (Tung and Winn, 2010; Kataoka et al., 2013), which is strongly relevant to postnatal autistic-like behaviors (Kataoka et al., 2013; Moldrich et al., 2013). VPA might interrupt the regulation in the upstream of the embryonic oxt gene expression during the critical window of developing brain. Additionally, VPA could also disturb Wnt (Wang et al., 2015; Qin et al., 2016) and GABAergic (Löscher, 2002; Olexova et al., 2016) signaling pathways which play important roles in the progress of cell proliferation and differentiation (Jung et al., 2008), as well as the establishment of neural networks (Baltz et al., 2010) in the developing brain. Previous studies have shown that rats prenatally exposed to VPA had fewer neurons in several brain regions (such as prefrontal and somatosensory cortices) during infancy and adolescence (Rodier et al., 1996; Kataoka et al., 2013), which may contribute to the lower brain-weight and thinner cortex of VPA rats (Mychasiuk et al., 2012; Favre et al., 2013). These may partially explain our findings that the number of OXT-ir cells in the hypothalamus of VPA rats were abnormally low.

It should be pointed out that central OXT dysfunction is not limited to the VPA-induced autistic model. In several models of monogenic or multigenic heritable forms of autism, central OXT levels were also reported to be dysregulated. These models included BTBR mice, and CNTNAP2, MAGEL2, and Oprm1 knockout (KO) mice. BTBR mice displayed reduced social interaction (Bales et al., 2014), accompanied with higher OXT content in the PVN of the hypothalamus (Silverman et al., 2010b). In contrast, the number of OXT-ir neurons in the PVN, as well as the level of OXT in the whole brain, were decreased in CNTNAP2 KO mice (Penagarikano et al., 2015). Similarly, lower OXT levels in nucleus accumbens (NAc) were reported in Oprm1 KO mice (Becker et al., 2014). MAGEL2 is believed to be involved in the pathogenesis of ASD. Previous studies have shown that OXT content in hypothalamus was significantly lower in newborn mice lacking MAGEL2 (Schaller et al., 2010). Conversely, in adult $M A G E L 2^{-/-}$mice, increased number of OXT-ir neurons and peptide levels were observed in the hypothalamus and hypophysis, respectively (Meziane et al., 2015). Although the above-mentioned autism models vary in etiology, they share a certain degree of impaired social behaviors associated with central OXT defects, suggesting dysregulation of OXT pathway may be the common cause of some of the social deficits.

Unexpectedly, our findings of lower OXT mRNA level in VPA rats were inconsistent with the results reported in a previous study, as they observed increased OXT mRNA levels in the PVN and SON of VPA rats (Stefanik et al., 2015). It should be noted that the VPA rats in that study were adults when OXT expression was measured, while the rats used in the present study were still in adolescence. Additionally, their analyses were under sex-mixed manner. Rats might experience a dramatically physiological change when transitioning from adolescence to adulthood. Sex hormones, like estrogen, secreted by adult rats could regulate oxt expression in the brain (Dellovade et al., 1999; Nomura et al., 2002; Murakami et al., 2011). These confounding factors may ultimately influence OXT expression.

Similar to the findings of a recent study (Hara et al., 2017), we also observed that a single intranasal OXT could restore the social preference deficits of VPA rats. The pro-social effects of nasal OXT manipulation have been confirmed in animal studies (Bales et al., 2014; Calcagnoli et al., 2015; Simpson et al., 2017) and clinical studies (Hollander et al., 2003; Andari et al., 2010; Watanabe et al., 2015; Yatawara et al., 2016). Although the underlying mechanism is unclear, immunohistochemical staining of c-Fos (Jia et al., 2008; Hara et al., 2017) and imaging studies (Bethlehem et al., 2013; Rilling et al., 2014) have shown that nasal administration of OXT activates social behavior-relevant areas in the brain. However, an acute exogenous OXT supplementation only produced a temporary pro-social effect (Penagarikano et al., 2015; Hara et al., 2017), probably because the half-life of OXT in the brain is relatively short (Jones and Robinson, 1982; Mens et al., 1983).

Thus, we report the first trial for the treatment of VPA rats with OXT at the neonatal stage. Results showed that neonatal OXT manipulation in the first week after birth improved the social preference and decreased stereotyped behavior in the VPA rats. We further found that the number of OXT-ir cells in the PVN was no longer abnormally low. This long-lasting improvement of postnatal OXT intervention on social behavior and OXT-ir neurons has also been observed in prairie voles (Bales and Carter, 2003; Yamamoto et al., 2004), mandarin voles (Jia et al., 2008), mice (Mogi et al., 2014), and monogenic mouse models of autism (Meziane et al., 2015; Penagarikano et al., 2015). Studies have suggested that OXT promotes neuron proliferation and differentiation. Incubation with OXT in vitro $(0.01 \mu \mathrm{M}$, on the 8th day of neural induction) improves the proliferation and neural differentiation of mouse adipose tissue-derived stem cells (ADSCs) (Jafarzadeh et al., 2014). Rodent studies have revealed that intraperitoneal $(1 \mathrm{mg} / \mathrm{kg}$ body weight) or hippocampal (1 ng) injections of OXT enhances neurogenesis in the adult hippocampus (Leuner et al., 2012; Sanchez-Vidana et al., 2016), and this is associated with activation of oxytocin receptors (Oxtr) expressed in CA3 pyramidal neurons (Lin et al., 2017). OXT binding to Oxtr induces the release of $\mathrm{Ca}^{2+}$ from intracellular stores (Gimpl and Fahrenholz, 2001; Noiseux et al., 2012), and the activation of intracellular $\mathrm{Ca}^{2}+$ signaling pathway can further promote cell differentiation and maturation (Batut et al., 2005; Leclerc et al., 2012). In rodents, Oxtr synthesis begins during the embryonic stage and mature Oxtr are expressed in the hypothalamus after birth (Yoshimura et al., 1996; Wang and Young, 1997). In the early stage of life, OXT neurons mainly express the Oxtr (without vasopressin receptors) and response to their own peptides (Freund-Mercier et al., 1994; Hurbin et al., 1998; Chevaleyre et al., 2000). Thus, postnatal OXT administration might activate the Oxtr on the OXT neurons, subsequently enhancing their proliferation. From these results, it is likely that amelioration in autistic behaviors was associated 
with the promotion of OXT neuronal development. Additionally, the c-Fos staining showed that a single intraperitoneal OXT injection given after birth activated the neurons in several brain areas, especially the SON of male prairie voles (Cushing et al., 2003). These data indicate that neonatal OXT played a role in modulating the activity of neurons in the developing brain resulting in remodeling of the neuronal circuit and modulating social behaviors.

The present study proved that early postnatal OXT manipulation had a long-term therapeutic effect on an environmental factor-induced autistic model. Early postnatal OXT treatment has also been effective for other monogenic models of autism. Further, previous findings have shown that OXT treatment was more effective in the early stage of life than in adolescence (Penagarikano et al., 2015). In clinical studies, OXT treatment for patients with ASD is mostly concentrated during adolescence or adulthood, with a demonstrable therapeutic effect (Hollander et al., 2003; Hollander et al., 2007; Andari et al., 2010; Guastella et al., 2010; Hall et al., 2012; Rilling et al., 2014). However, the long-term efficacy of multiple or chronic OXT treatment is controversial (Tachibana et al., 2013; Guastella et al., 2015; Watanabe et al., 2015). Combining the findings regarding postnatal OXT treatment in different types of autistic models, starting the treatment at an early age might be advantageous. Further research is required for underlying mechanism that links

\section{REFERENCES}

Andari, E., Duhamel, J. R., Zalla, T., Herbrecht, E., Leboyer, M., and Sirigu, A. (2010). Promoting social behavior with oxytocin in high-functioning autism spectrum disorders. Proc. Natl. Acad. Sci. U.S.A. 107, 4389-4394. doi: 10.1073/ pnas.0910249107

Ashwood, P., and Van de Water, J. (2004). A review of autism and the immune response. Clin. Dev. Immunol. 11, 165-174. doi: 10.1080/ 10446670410001722096

Bales, K. L., and Carter, C. S. (2003). Developmental exposure to oxytocin facilitates partner preferences in male prairie voles (Microtus ochrogaster). Behav. Neurosci. 117, 854-859. doi: 10.1037/0735-7044.117.4.854

Bales, K. L., Solomon, M., Jacob, S., Crawley, J. N., Silverman, J. L., Larke, R. H., et al. (2014). Long-term exposure to intranasal oxytocin in a mouse autism model. Transl. Psychiatry 4:e480. doi: 10.1038/tp.2014.117

Bales, K. L., van Westerhuyzen, J. A., Lewis-Reese, A. D., Grotte, N. D., Lanter, J. A., and Carter, C. S. (2007). Oxytocin has dose-dependent developmental effects on pair-bonding and alloparental care in female prairie voles. Horm. Behav. 52, 274-279. doi: 10.1016/j.yhbeh.2007.05.004

Baltz, T., de Lima, A. D., and Voigt, T. (2010). Contribution of GABAergic interneurons to the development of spontaneous activity patterns in cultured neocortical networks. Front. Cell. Neurosci. 4:15. doi: 10.3389/fncel.2010.00015

Batut, J., Vandel, L., Leclerc, C., Daguzan, C., Moreau, M., and Neant, I. (2005). The $\mathrm{Ca}^{+}{ }^{+}$-induced methyltransferase xPRMT1b controls neural fate in amphibian embryo. Proc. Natl. Acad. Sci. U.S.A. 102, 15128-15133. doi: 10.1073/pnas. 0502483102

Becker, J. A., Clesse, D., Spiegelhalter, C., Schwab, Y., Le Merrer, J., and Kieffer, B. L. (2014). Autistic-like syndrome in mu opioid receptor null mice is relieved by facilitated mGluR4 activity. Neuropsychopharmacology 39, 2049-2060. doi: 10.1038/npp.2014.59

Benelli, A., Bertolini, A., Poggioli, R., Menozzi, B., Basaglia, R., and Arletti, R. (1995). Polymodal dose-response curve for oxytocin in the social recognition test. Neuropeptides 28, 251-255. doi: 10.1016/0143-4179(95)90029-2

Bertelsen, F., Moller, A., Folloni, D., Drasbek, K. R., Scheel-Kruger, J., and Landau, A. M. (2017). Increased GABAA receptor binding in amygdala after prenatal
OXT and ASD, as well as the selection of timing, duration, and route of administration for OXT treatment.

In summary, the present study provides some insights on the VPA-induced autistic model in rat, and showed that the early postnatal OXT treatment had a long-term therapeutic effects on autistic-like behaviors in these rats.

\section{AUTHOR CONTRIBUTIONS}

YD and HZ conceived and designed the study, performed the experiments, analyzed the data, and wrote the manuscript. RZ, $\mathrm{JH}$, and $\mathrm{SH}$ helped to design the study and contributed to analysis with constructive discussions. $\mathrm{RZ}, \mathrm{JH}, \mathrm{SH}, \mathrm{TB}$, and MS reviewed and edited the manuscript and made valuable suggestions. All authors approved the final version.

\section{FUNDING}

This work was supported by grants from the University of Ulm-Peking University Health Science Center Joint Center for Neuroscience Fund (BMU20160563) and the National Key Research and Development Program of China (2016YFC0105501).

administration of valproic acid to rats. Acta Neuropsychiatr. 29, 309-314 doi: 10.1017/neu.2016.59

Bethlehem, R. A., van Honk, J., Auyeung, B., and Baron-Cohen, S. (2013). Oxytocin, brain physiology, and functional connectivity: a review of intranasal oxytocin fMRI studies. Psychoneuroendocrinology 38, 962-974. doi: 10.1016/j. psyneuen.2012.10.011

Bhat, S., Acharya, U. R., Adeli, H., Bairy, G. M., and Adeli, A. (2014). Autism: cause factors, early diagnosis and therapies. Rev. Neurosci. 25, 841-850. doi: 10.1515/revneuro-2014-0056

Bowers, K., Lin, P.-I., and Erickson, C. (2015). Pharmacogenomic medicine in autism: challenges and opportunities. Pediatr. Drugs 17, 115-124. doi: 10.1007/ s40272-014-0106-0

Bromley, R. L., Mawer, G., Clayton-Smith, J., and Baker, G. A. (2008). Autism spectrum disorders following in utero exposure to antiepileptic drugs. Neurology 71, 1923-1924. doi: 10.1212/01.wnl.0000339399.64213.1a

Bromley, R. L., Mawer, G. E., Briggs, M., Cheyne, C., Clayton-Smith, J., GarciaFinana, M., et al. (2013). The prevalence of neurodevelopmental disorders in children prenatally exposed to antiepileptic drugs. J. Neurol. Neurosurg. Psychiatry 84, 637-643. doi: 10.1136/jnnp-2012-304270

Brown, A. S. (2012). Epidemiologic studies of exposure to prenatal infection and risk of schizophrenia and autism. Dev. Neurobiol. 72, 1272-1276. doi: 10.1002/ dneu. 22024

Calcagnoli, F., Kreutzmann, J. C., de Boer, S. F., Althaus, M., and Koolhaas, J. M. (2015). Acute and repeated intranasal oxytocin administration exerts antiaggressive and pro-affiliative effects in male rats. Psychoneuroendocrinology 51, 112-121. doi: 10.1016/j.psyneuen.2014.09.019

Caldwell, H. K. (2017). Oxytocin and vasopressin: powerful regulators of social behavior. Neuroscientist 23, 517-528. doi: 10.1177/1073858417708284

Chaste, P., and Leboyer, M. (2012). Autism risk factors: genes, environment, and gene-environment interactions. Dialogues Clin. Neurosci. 14, 281-292.

Chevaleyre, V., Dayanithi, G., Moos, F. C., and Desarmenien, M. G. (2000). Developmental regulation of a local positive autocontrol of supraoptic neurons. J. Neurosci. 20, 5813-5819. doi: 10.1523/JNEUROSCI.20-15-05813.2000

Christensen, J., Gronborg, T. K., Sorensen, M. J., Schendel, D., Parner, E. T., Pedersen, L. H., et al. (2013). Prenatal valproate exposure and risk of autism 
spectrum disorders and childhood autism. JAMA 309, 1696-1703. doi: 10.1001/ jama.2013.2270

Constantino, J. N., and Charman, T. (2012). Gender bias, female resilience, and the sex ratio in autism. J. Am. Acad. Child Adolesc. Psychiatry 51, 756-758. doi: 10.1016/j.jaac.2012.05.017

Cservenak, M., Keller, D., Kis, V., Fazekas, E. A., Ollos, H., Leko, A. H., et al. (2017). A thalamo-hypothalamic pathway that activates oxytocin neurons in social contexts in female rats. Endocrinology 158, 335-348.

Cushing, B. S., Yamamoto, Y., Hoffman, G. E., and Carter, C. S. (2003). Central expression of c-Fos in neonatal male and female prairie voles in response to treatment with oxytocin. Dev. Brain Res. 143, 129-136. doi: 10.1016/S01653806(03)00105-6

Dadds, M. R., MacDonald, E., Cauchi, A., Williams, K., Levy, F., and Brennan, J. (2014). Nasal oxytocin for social deficits in childhood autism: a randomized controlled trial. J. Autism Dev. Disord. 44, 521-531. doi: 10.1007/s10803-0131899-3

De Rubeis, S., He, X., Goldberg, A. P., Poultney, C. S., Samocha, K., Cicek, A. E., et al. (2014). Synaptic, transcriptional and chromatin genes disrupted in autism. Nature 515, 209-215. doi: 10.1038/nature13772

Dellovade, T. L., Zhu, Y.-S., and Pfaff, D. W. (1999). Thyroid hormones and estrogen affect oxytocin gene expression in hypothalamic neurons. J. Neuroendocrinol. 11, 1-10. doi: 10.1046/j.1365-2826.1999.00250.x

Favre, M. R., Barkat, T. R., Lamendola, D., Khazen, G., Markram, H., and Markram, K. (2013). General developmental health in the VPA-rat model of autism. Front. Behav. Neurosci. 7:88. doi: 10.3389/fnbeh.2013.00088

Ferguson, J. N., Aldag, J. M., Insel, T. R., and Young, L. J. (2001). Oxytocin in the medial amygdala is essential for social recognition in the mouse. J. Neurosci. 21, 8278-8285. doi: 10.1523/JNEUROSCI.21-20-08278.2001

Ferguson, J. N., Young, L. J., Hearn, E. F., Matzuk, M. M., Insel, T. R., and Winslow, J. T. (2000). Social amnesia in mice lacking the oxytocin gene. Nat. Genet. 25, 284-288. doi: 10.1038/77040

Freund-Mercier, M. J., Stoeckel, M. E., and Klein, M. J. (1994). Oxytocin receptors on oxytocin neurones: histoautoradiographic detection in the lactating rat. J. Physiol. 480(Pt 1), 155-161. doi: 10.1113/jphysiol.1994.sp020349

Gigliucci, V., Leonzino, M., Busnelli, M., Luchetti, A., Palladino, V.S., D’Amato, F.R., et al. (2014). Region specific up-regulation of oxytocin receptors in the opioid Oprm1-/- mouse model of autism. Front. Pediatr. 2:91. doi: 10.3389/ fped.2014.00091

Gimpl, G., and Fahrenholz, F. (2001). The oxytocin receptor system: structure, function, and regulation. Physiol. Rev. 81, 629-683. doi: 10.1152/physrev.2001. 81.2.629

Gottlicher, M., Minucci, S., Zhu, P., Kramer, O. H., Schimpf, A., Giavara, S., et al. (2001). Valproic acid defines a novel class of HDAC inhibitors inducing differentiation of transformed cells. EMBO J. 20, 6969-6978. doi: 10.1093/ emboj/20.24.6969

Guastella, A. J., Einfeld, S. L., Gray, K. M., Rinehart, N. J., Tonge, B. J., Lambert, T. J., et al. (2010). Intranasal oxytocin improves emotion recognition for youth with autism spectrum disorders. Biol. Psychiatry 67, 692-694. doi: 10.1016/j. biopsych.2009.09.020

Guastella, A. J., Gray, K. M., Rinehart, N. J., Alvares, G. A., Tonge, B. J., Hickie, I. B., et al. (2015). The effects of a course of intranasal oxytocin on social behaviors in youth diagnosed with autism spectrum disorders: a randomized controlled trial. J. Child Psychol. Psychiatr. 56, 444-452. doi: 10.1111/jcpp.12305

Hall, S. S., Lightbody, A. A., McCarthy, B. E., Parker, K. J., and Reiss, A. L. (2012). Effects of intranasal oxytocin on social anxiety in males with fragile $\mathrm{X}$ syndrome. Psychoneuroendocrinology 37, 509-518. doi: 10.1016/j.psyneuen.2011.07.020

Hallmayer, J., Cleveland, S., Torres, A., et al. (2011). Genetic heritability and shared environmental factors among twin pairs with autism. Arch. Gen. Psychiatry 68, 1095-1102. doi: 10.1001/archgenpsychiatry.2011.76

Hara, Y., Ago, Y., Higuchi, M., Hasebe, S., Nakazawa, T., Hashimoto, H., et al. (2017). Oxytocin attenuates deficits in social interaction but not recognition memory in a prenatal valproic acid-induced mouse model of autism. Horm. Behav. 96, 130-136. doi: 10.1016/j.yhbeh.2017.09.013

Hollander, E., Bartz, J., Chaplin, W., Phillips, A., Sumner, J., Soorya, L., et al. (2007). Oxytocin increases retention of social cognition in autism. Biol. Psychiatry 61, 498-503. doi: 10.1016/j.biopsych.2006.05.030

Hollander, E., Novotny, S., Hanratty, M., Yaffe, R., DeCaria, C. M., Aronowitz, B. R., et al. (2003). Oxytocin infusion reduces repetitive behaviors in adults with autistic and Asperger's disorders. Neuropsychopharmacology 28, 193-198. doi: 10.1038/sj.npp.1300021

Hung, L. W., Neuner, S., Polepalli, J. S., Beier, K. T., Wright, M., Walsh, J. J., et al. (2017). Gating of social reward by oxytocin in the ventral tegmental area. Science 357, 1406-1411. doi: 10.1126/science.aan4994

Hurbin, A., Boissin-Agasse, L., Orcel, H., Rabie, A., Joux, N., Desarmenien, M. G., et al. (1998). The V1a and V1b, but not V2, vasopressin receptor genes are expressed in the supraoptic nucleus of the rat hypothalamus, and the transcripts are essentially colocalized in the vasopressinergic magnocellular neurons. Endocrinology 139, 4701-4707. doi: 10.1210/endo.139.11.6320

Ivanov, H. Y., Stoyanova, V. K., Popov, N. T., and Vachev, T. I. (2015). Autism spectrum disorder - a complex genetic disorder. Folia Med. 57, 19-28. doi: 10.1515/folmed-2015-0015

Jafarzadeh, N., Javeri, A., Khaleghi, M., and Taha, M. F. (2014). Oxytocin improves proliferation and neural differentiation of adipose tissue-derived stem cells. Neurosci. Lett. 564, 105-110. doi: 10.1016/j.neulet.2014.02.012

Jia, R., Tai, F., An, S., Broders, H., and Sun, R. (2008). Neonatal manipulation of oxytocin influences the partner preference in mandarin voles (Microtus mandarinus). Neuropeptides 42, 525-533. doi: 10.1016/j.npep.2008.06.001

Jin, D., Liu, H.-X., Hirai, H., Torashima, T., Nagai, T., Lopatina, O., et al. (2007). $\mathrm{CD} 38$ is critical for social behaviour by regulating oxytocin secretion. Nature 446, 41-45. doi: $10.1038 /$ nature 05526

Jones, P. M., and Robinson, I. C. (1982). Differential clearance of neurophysin and neurohypophysial peptides from the cerebrospinal fluid in conscious guinea pigs. Neuroendocrinology 34, 297-302. doi: 10.1159/000123316

Jung, G. A., Yoon, J. Y., Moon, B. S., Yang, D. H., Kim, H. Y., Lee, S. H., et al. (2008). Valproic acid induces differentiation and inhibition of proliferation in neural progenitor cells via the beta-catenin-Ras-ERK-p21Cip/WAF1 pathway. BMC Cell Biol. 9:66. doi: 10.1186/1471-2121-9-66

Kalueff, A. V., Stewart, A. M., Song, C., Berridge, K. C., Graybiel, A. M., and Fentress, J. C. (2016). Neurobiology of rodent self-grooming and its value for translational neuroscience. Nat. Rev. Neurosci. 17, 45-59. doi: 10.1038/nrn. 2015.8

Kataoka, S., Takuma, K., Hara, Y., Maeda, Y., Ago, Y., and Matsuda, T. (2013). Autism-like behaviours with transient histone hyperacetylation in mice treated prenatally with valproic acid. Int. J. Neuropsychopharmacol. 16, 91-103. doi: $10.1017 /$ s1461145711001714

Klei, L., Sanders, S. J., Murtha, M. T., Hus, V., Lowe, J. K., Willsey, A. J., et al. (2012). Common genetic variants, acting additively, are a major source of risk for autism. Mol. Autism 3:9. doi: 10.1186/2040-2392-3-9

Kosaka, H., Munesue, T., Ishitobi, M., Asano, M., Omori, M., Sato, M., et al. (2012). Long-term oxytocin administration improves social behaviors in a girl with autistic disorder. BMC Psychiatry 12:110. doi: 10.1186/1471-244X-12-110

Leclerc, C., Neant, I., and Moreau, M. (2012). The calcium: an early signal that initiates the formation of the nervous system during embryogenesis. Front. Mol. Neurosci. 5:3. doi: 10.3389/fnmol.2012.00064

Leuner, B., Caponiti, J. M., and Gould, E. (2012). Oxytocin stimulates adult neurogenesis even under conditions of stress and elevated glucocorticoids. Hippocampus 22, 861-868. doi: 10.1002/hipo.20947

Lin, Y. T., Chen, C. C., Huang, C. C., Nishimori, K., and Hsu, K. S. (2017). Oxytocin stimulates hippocampal neurogenesis via oxytocin receptor expressed in CA3 pyramidal neurons. Nat. Commun. 8:537. doi: 10.1038/s41467-017-00675-5

Löscher, W. (2002). Basic pharmacology of valproate: a review after 35 years of clinical use for the treatment of epilepsy. CNS Drugs 16, 669-694. doi: 10.2165/ 00023210-200216100-00003

Lukas, M., and Neumann, I. D. (2012). Nasal application of neuropeptide S reduces anxiety and prolongs memory in rats: social versus non-social effects. Neuropharmacology 62, 398-405. doi: 10.1016/j.neuropharm.2011.08.016

Lukas, M., Toth, I., Reber, S. O., Slattery, D. A., Veenema, A. H., and Neumann, I. D. (2011). The neuropeptide oxytocin facilitates pro-social behavior and prevents social avoidance in rats and mice. Neuropsychopharmacology 36, 2159-2168. doi: 10.1038/npp.2011.95

MacDonald, R., Parry-Cruwys, D., Dupere, S., and Ahearn, W. (2014). Assessing progress and outcome of early intensive behavioral intervention for toddlers with autism. Res. Dev. Disabil. 35, 3632-3644. doi: 10.1016/j.ridd.2014.08.036

McCracken, J. T., McGough, J., Shah, B., Cronin, P., Hong, D., Aman, M. G., et al. (2002). Risperidone in children with autism and serious behavioral problems. N. Engl. J. Med. 347, 314-321. doi: 10.1056/NEJMoa013171 
Meador, K. J., Baker, G. A., Browning, N., Cohen, M. J., Bromley, R. L., ClaytonSmith, J., et al. (2013). Fetal antiepileptic drug exposure and cognitive outcomes at age 6 years (NEAD study): a prospective observational study. Lancet Neurol. 12, 244-252. doi: 10.1016/s1474-4422(12)70323-x

Mens, W. B. J., Witter, A., and Greidanus, T. B. V. (1983). Penetration of neurohypophyseal hormones from plasma into cerebrospinal-fluid (CSF) half-times of disappearance of these neuropeptides from CSF. Brain Res. 262, 143-149. doi: 10.1016/0006-8993(83)90478-X

Meziane, H., Schaller, F., Bauer, S., Villard, C., Matarazzo, V., Riet, F., et al. (2015). An early postnatal oxytocin treatment prevents social and learning deficits in adult mice deficient for magel2, a gene involved in Prader-Willi syndrome and autism. Biol. Psychiatry 78, 85-94. doi: 10.1016/j.biopsych.2014.11.010

Mogi, K., Ooyama, R., Nagasawa, M., and Kikusui, T. (2014). Effects of neonatal oxytocin manipulation on development of social behaviors in mice. Physiol. Behav. 133, 68-75. doi: 10.1016/j.physbeh.2014.05.010

Moldrich, R. X., Leanage, G., She, D., Dolan-Evans, E., Nelson, M., Reza, N., et al. (2013). Inhibition of histone deacetylase in utero causes sociability deficits in postnatal mice. Behav. Brain Res. 257, 253-264. doi: 10.1016/j.bbr.2013.09.049

Murakami, G., Hunter, R. G., Fontaine, C., Ribeiro, A., and Pfaff, D. (2011). Relationships among estrogen receptor, oxytocin and vasopressin gene expression and social interaction in male mice. Eur. J. Neurosci. 34, 469-477. doi: 10.1111/j.1460-9568.2011.07761.x

Mychasiuk, R., Richards, S., Nakahashi, A., Kolb, B., and Gibb, R. (2012). Effects of rat prenatal exposure to valproic acid on behaviour and neuro-anatomy. Dev. Neurosci. 34, 268-276. doi: 10.1159/000341786

Neumann, I. D., Maloumby, R., Beiderbeck, D. I., Lukas, M., and Landgraf, R. (2013). Increased brain and plasma oxytocin after nasal and peripheral administration in rats and mice. Psychoneuroendocrinology 38, 1985-1993. doi: 10.1016/j.psyneuen.2013.03.003

Nicolini, C., and Fahnestock, M. (2018). The valproic acid-induced rodent model of autism. Exp. Neurol. 299(Pt A), 217-227. doi: 10.1016/j.expneurol.2017.04.017

Noiseux, N., Borie, M., Desnoyers, A., Menaouar, A., Stevens, L. M., Mansour, S., et al. (2012). Preconditioning of stem cells by oxytocin to improve their therapeutic potential. Endocrinology 153, 5361-5372. doi: 10.1210/en.20121402

Nomura, M., McKenna, E., Korach, K. S., Pfaff, D. W., and Ogawa, S. (2002). Estrogen receptor-beta regulates transcript levels for oxytocin and arginine vasopressin in the hypothalamic paraventricular nucleus of male mice. Mol. Brain Res. 109, 84-94. doi: 10.1016/S0169-328X(02)00525-9

Oettl, L. L., Ravi, N., Schneider, M., Scheller, M. F., Schneider, P., Mitre, M., et al. (2016). Oxytocin enhances social recognition by modulating cortical control of early olfactory processing. Neuron 90, 609-621. doi: 10.1016/j.neuron.2016.03. 033

Olexova, L., Stefanik, P., and Krskova, L. (2016). Increased anxiety-like behaviour and altered GABAergic system in the amygdala and cerebellum of VPA rats - an animal model of autism. Neurosci. Lett. 629, 9-14. doi: 10.1016/j.neulet.2016.06. 035

Ornoy, A. (2009). Valproic acid in pregnancy: how much are we endangering the embryo and fetus? Reprod. Toxicol. 28, 1-10. doi: 10.1016/j.reprotox.2009.02. 014

Penagarikano, O., Lazaro, M.T., Lu, X.H., Gordon, A., Dong, H., Lam, H.A., et al. (2015). Exogenous and evoked oxytocin restores social behavior in the Cntnap2 mouse model of autism. Sci. Transl. Med. 7:271ra8. doi: 10.1126/scitranslmed. 3010257

Pobbe, R. L. H., Pearson, B. L., Blanchard, D. C., and Blanchard, R. J. (2012a). Oxytocin receptor and Mecp2308/Y knockout mice exhibit altered expression of autism-related social behaviors. Physiol. Behav. 107, 641-648. doi: 10.1016/j. physbeh.2012.02.024

Pobbe, R. L. H., Pearson, B. L., Defensor, E. B., Bolivar, V. J., Young, W. S., Lee, H.-J., et al. (2012b). Oxytocin receptor knockout mice display deficits in the expression of autism-related behaviors. Horm. Behav. 61, 436-444. doi: 10.1016/ j.yhbeh.2011.10.010

Popik, P., and van Ree, J. M. (1991). Oxytocin but not vasopressin facilitates social recognition following injection into the medial preoptic area of the rat brain. Eur. Neuropsychopharmacol. 1, 555-560. doi: 10.1016/0924-977X(91)90010-R

Preti, A., Melis, M., Siddi, S., Vellante, M., Doneddu, G., and Fadda, R. (2014). Oxytocin and autism: a systematic review of randomized controlled trials. J. Child Adolesc. Psychopharmacol. 24, 54-68. doi: 10.1089/cap.2013.0040
Qin, L., Dai, X., and Yin, Y. (2016). Valproic acid exposure sequentially activates Wnt and mTOR pathways in rats. Mol. Cell. Neurosci. 75, 27-35. doi: 10.1016/j. mcn.2016.06.004

Raam, T., McAvoy, K. M., Besnard, A., Veenema, A., and Sahay, A. (2017). Hippocampal oxytocin receptors are necessary for discrimination of social stimuli. Nat. Commun. 8:2001. doi: 10.1038/s41467-017-02173-0

Rasalam, A. D., Hailey, H., Williams, J. H., Moore, S. J., Turnpenny, P. D., Lloyd, D. J., et al. (2005). Characteristics of fetal anticonvulsant syndrome associated autistic disorder. Dev. Med. Child Neurol. 47, 551-555. doi: 10.1111/j.14698749.2005.tb01190.x

Rilling, J. K., Demarco, A. C., Hackett, P. D., Chen, X., Gautam, P., Stair, S., et al. (2014). Sex differences in the neural and behavioral response to intranasal oxytocin and vasopressin during human social interaction. Psychoneuroendocrinology 39, 237-248. doi: 10.1016/j.psyneuen.2013.09.022

Rodier, P. M., Ingram, J. L., Tisdale, B., Nelson, S., and Romano, J. (1996). Embryological origin for autism: developmental anomalies of the cranial nerve motor nuclei. J. Comp. Neurol. 370, 247-261. doi: 10.1002/(SICI)10969861(19960624)370:2<247::AID-CNE8>3.0.CO;2-2

Ross, H. E., Cole, C. D., Smith, Y., Neumann, I. D., Landgraf, R., Murphy, A. Z., et al. (2009). Characterization of the oxytocin system regulating affiliative behavior in female prairie voles. Neuroscience 162, 892-903. doi: 10.1016/j. neuroscience.2009.05.055

Roullet, F. I., Lai, J. K. Y., and Foster, J. A. (2013). In utero exposure to valproic acid and autism - A current review of clinical and animal studies. Neurotoxicol. Teratol. 36, 47-56. doi: 10.1016/j.ntt.2013.01.004

Sala, M., Braida, D., Lentini, D., Busnelli, M., Bulgheroni, E., Capurro, V., et al. (2011). Pharmacologic rescue of impaired cognitive flexibility, social deficits, increased aggression, and seizure susceptibility in oxytocin receptor null mice: a neurobehavioral model of autism. Biol. Psychiatry 69, 875-882. doi: 10.1016/j. biopsych.2010.12.022

Sanchez-Vidana, D. I., Chan, N. M., Chan, A. H., Hui, K. K., Lee, S., Chan, H. Y., et al. (2016). Repeated treatment with oxytocin promotes hippocampal cell proliferation, dendritic maturation and affects socio-emotional behavior. Neuroscience 333, 65-77. doi: 10.1016/j.neuroscience.2016.07.005

Schaller, F., Watrin, F., Sturny, R., Massacrier, A., Szepetowski, P., and Muscatelli, F. (2010). A single postnatal injection of oxytocin rescues the lethal feeding behaviour in mouse newborns deficient for the imprinted Magel2 gene. Hum. Mol. Genet. 19, 4895-4905. doi: 10.1093/hmg/ddq424

Schneider, T., and Przewlocki, R. (2005). Behavioral alterations in rats prenatally exposed to valproic acid: animal model of autism. Neuropsychopharmacology 30, 80-89. doi: 10.1038/sj.npp.1300518

Sebat, J., Lakshmi, B., Malhotra, D., Troge, J., Lese-Martin, C., Walsh, T., et al. (2007). Strong association of de novo copy number mutations with autism. Science 316, 445-449. doi: 10.1126/science.1138659

Silverman, J. L., Yang, M., Lord, C., and Crawley, J. N. (2010a). Behavioural phenotyping assays for mouse models of autism. Nat. Rev. Neurosci. 11, 490502. doi: $10.1038 / \mathrm{nrn} 2851$

Silverman, J. L., Yang, M., Turner, S. M., Katz, A. M., Bell, D. B., Koenig, J. I., et al. (2010b). Low stress reactivity and neuroendocrine factors in the BTBR $\mathrm{T}^{+}$Tf/J mouse model of autism. Neuroscience 171, 1197-1208. doi: 10.1016/j. neuroscience.2010.09.059

Simpson, E. A., Paukner, A., Sclafani, V., Kaburu, S. S. K., Suomi, S. J., and Ferrari, P. F. (2017). Acute oxytocin improves memory and gaze following in male but not female nursery-reared infant macaques. Psychopharmacology 234, 497-506. doi: $10.1007 / \mathrm{s} 00213-016-4480-\mathrm{x}$

Smith, C. J. W., Mogavero, J. N., Tulimieri, M. T., and Veenema, A. H. (2017). Involvement of the oxytocin system in the nucleus accumbens in the regulation of juvenile social novelty-seeking behavior. Horm. Behav. 93, 94-98. doi: 10. 1016/j.yhbeh.2017.05.005

Smith, V., and Brown, N. (2014). Prenatal valproate exposure and risk of autism spectrum disorders and childhood autism. Arch. Dis. Child Educ. Pract. Ed. 99:198. doi: 10.1136/archdischild-2013-305636

Song, Z., and Albers, H. E. (2017). Cross-talk among oxytocin and argininevasopressin receptors: relevance for basic and clinical studies of the brain and periphery. Front. Neuroendocrinol. doi: 10.1016/j.yfrne.2017.10.004 [Epub ahead of print].

Stefanik, P., Olexova, L., and Krskova, L. (2015). Increased sociability and gene expression of oxytocin and its receptor in the brains of rats affected prenatally 
by valproic acid. Pharmacol. Biochem. Behav. 131, 42-50. doi: 10.1016/j.pbb. 2015.01.021

Stoop, R. (2012). Neuromodulation by oxytocin and vasopressin. Neuron 76, 142-159. doi: 10.1016/j.neuron.2012.09.025

Tachibana, M., Kagitani-Shimono, K., Mohri, I., Yamamoto, T., Sanefuji, W., Nakamura, A., et al. (2013). Long-term administration of intranasal oxytocin is a safe and promising therapy for early adolescent boys with autism spectrum disorders. J. Child Adolesc. Psychopharmacol. 23, 123-127. doi: 10.1089/cap. 2012.0048

Teng, B. L., Nikolova, V. D., Riddick, N. V., Agster, K. L., Crowley, J. J., Baker, L. K., et al. (2016). Reversal of social deficits by subchronic oxytocin in two autism mouse models. Neuropharmacology 105, 61-71. doi: 10.1016/j.neuropharm. 2015.12.025

Tonge, B. J., Bull, K., Brereton, A., and Wilson, R. (2014). A review of evidencebased early intervention for behavioural problems in children with autism spectrum disorder: the core components of effective programs, child-focused interventions and comprehensive treatment models. Curr. Opin. Psychiatry 27, 158-165. doi: 10.1097/yco.0000000000000043

Tordjman, S., Somogyi, E., Coulon, N., Kermarrec, S., Cohen, D., Bronsard, G., et al. (2014). Gene? $\times$ ?environment interactions in autism spectrum disorders: role of epigenetic mechanisms. Front. Psychiatry 5:53. doi: 10.3389/fpsyt.2014. 00053

Tung, E. W., and Winn, L. M. (2010). Epigenetic modifications in valproic acidinduced teratogenesis. Toxicol. Appl. Pharmacol. 248, 201-209. doi: 10.1016/j. taap.2010.08.001

van der Kooij, M. A., and Sandi, C. (2012). Social memories in rodents: methods, mechanisms and modulation by stress. Neurosci. Biobehav. Rev. 36, 1763-1772. doi: 10.1016/j.neubiorev.2011.10.006

VanderLaan, D. P., Leef, J. H., Wood, H., Hughes, S. K., and Zucker, K. J. (2015). Autism spectrum disorder risk factors and autistic traits in gender dysphoric children. J. Autism Dev. Disord. 45, 1742-1750. doi: 10.1007/s10803-014-23313

Virués-Ortega, J. (2010). Applied behavior analytic intervention for autism in early childhood: meta-analysis, meta-regression and dose-response meta-analysis of multiple outcomes. Clin. Psychol. Rev. 30, 387-399. doi: 10.1016/j.cpr.2010.01. 008

Wang, L., Liu, Y., Li, S., Long, Z. Y., and Wu, Y. M. (2015). Wnt signaling pathway participates in valproic acid-induced neuronal differentiation of neural stem cells. Int. J. Clin. Exp. Pathol. 8, 578-585.

Wang, Z., and Young, L. J. (1997). Ontogeny of oxytocin and vasopressin receptor binding in the lateral septum in prairie and montane voles. Dev. Brain Res. 104, 191-195. doi: 10.1016/S0165-3806(97)00138-7

Watanabe, T., Kuroda, M., Kuwabara, H., Aoki, Y., Iwashiro, N., Tatsunobu, N., et al. (2015). Clinical and neural effects of six-week administration of oxytocin on core symptoms of autism. Brain 138, 3400-3412. doi: 10.1093/brain/awv249
Wu, H., Wang, X., Gao, J., Liang, S., Hao, Y., Sun, C., et al. (2017). Fingolimod (FTY720) attenuates social deficits, learning and memory impairments, neuronal loss and neuroinflammation in the rat model of autism. Life Sci. 173, 43-54. doi: 10.1016/j.lfs.2017.01.012

Xu, X. J., Zhang, H. F., Shou, X. J., Li, J., Jing, W. L., Zhou, Y., et al. (2015). Prenatal hyperandrogenic environment induced autistic-like behavior in rat offspring. Physiol. Behav. 138, 13-20. doi: 10.1016/j.physbeh.2014. 09.014

Yamaguchi, H., Hara, Y., Ago, Y., Takano, E., Hasebe, S., Nakazawa, T., et al. (2017). Environmental enrichment attenuates behavioral abnormalities in valproic acid-exposed autism model mice. Behav. Brain Res. 333, 67-73. doi: 10.1016/ j.bbr.2017.06.035

Yamamoto, Y., Cushing, B. S., Kramer, K. M., Epperson, P. D., Hoffman, G. E., and Carter, C. S. (2004). Neonatal manipulations of oxytocin alter expression of oxytocin and vasopressin immunoreactive cells in the paraventricular nucleus of the hypothalamus in a gender-specific manner. Neuroscience 125, 947-955. doi: 10.1016/j.neuroscience.2004.02.028

Yatawara, C. J., Einfeld, S. L., Hickie, I. B., Davenport, T. A., and Guastella, A. J. (2016). The effect of oxytocin nasal spray on social interaction deficits observed in young children with autism: a randomized clinical crossover trial. Mol. Psychiatry 21, 1225-1231. doi: 10.1038/mp.2015.162

Yoshihara, C., Numan, M., and Kuroda, K. O. (2017). Oxytocin and Parental Behaviors. Curr. Top. Behav. Neurosci.35, 119-153. doi: 10.1007/7854_2017_11

Yoshimura, R., Kimura, T., Watanabe, D., and Kiyama, H. (1996). Differential expression of oxytocin receptor mRNA in the developing rat brain. Neurosci. Res. 24, 291-304. doi: 10.1016/0168-0102(95)01003-3

Zhang, H. F., Li, H. X., Dai, Y. C., Xu, X. J., Han, S. P., Zhang, R., et al. (2015). Electro-acupuncture improves the social interaction behavior of rats. Physiol. Behav. 151, 485-493. doi: 10.1016/j.physbeh.2015.08.014

Zhang, R., Xu, X. J., Zhang, H. F., Han, S. P., and Han, J. S. (2017). The role of the oxytocin/arginine vasopressin system in animal models of autism spectrum disorder. Adv. Anat. Embryol. Cell Biol. 224, 135-158. doi: 10.1007/978-3-31952498-6_8

Conflict of Interest Statement: The authors declare that the research was conducted in the absence of any commercial or financial relationships that could be construed as a potential conflict of interest.

Copyright (c) 2018 Dai, Zhang, Schön, Böckers, Han, Han and Zhang. This is an open-access article distributed under the terms of the Creative Commons Attribution License (CC BY). The use, distribution or reproduction in other forums is permitted, provided the original author(s) and the copyright owner(s) are credited and that the original publication in this journal is cited, in accordance with accepted academic practice. No use, distribution or reproduction is permitted which does not comply with these terms. 